\title{
Aeroelastic stability of idling wind turbines
}

\author{
Kai Wang ${ }^{1}$, Vasilis A. Riziotis ${ }^{2}$, and Spyros G. Voutsinas ${ }^{2}$ \\ ${ }^{1}$ China-EU Institute for Clean and Renewable Energy, Huazhong University of Science and Technology, \\ 1037 Luoyu Rd, Wuhan, China \\ ${ }^{2}$ School of Mechanical Engineering, National Technical University of Athens, 15780 Athens, Greece \\ Correspondence to: Vasilis A. Riziotis (vasilis@ fluid.mech.ntua.gr)
}

Received: 15 December 2016 - Discussion started: 23 December 2016

Revised: 15 May 2017 - Accepted: 19 June 2017 - Published: 4 August 2017

\begin{abstract}
Wind turbine rotors in idling operation mode can experience high angles of attack within the poststall region that are capable of triggering stall-induced vibrations. The aim of the present paper is to extend the existing knowledge on the dynamics and aerodynamics of an idling wind turbine and characterize its stability. Rotor stability in slow idling operation is assessed on the basis of nonlinear time domain and linear eigenvalue analyses. The aim is to establish when linear analysis is reliable and identify cases for which nonlinear effects are significant. Analysis is performed for a $10 \mathrm{MW}$ conceptual wind turbine designed by DTU. First, the flow conditions that are likely to favor stall-induced instabilities are identified through nonlinear time domain aeroelastic simulations. Next, for the above specified conditions, eigenvalue stability analysis is performed to identify the low damped modes of the turbine. The eigenvalue stability results are evaluated through computations of the work done by the aerodynamic forces under imposed harmonic motion following the shape and frequency of the various modes. Nonlinear work characteristics predicted by the ONERA and Beddoes-Leishman (BL) dynamic stall models are compared. Both the eigenvalue and work analyses indicate that the asymmetric and symmetric out-of-plane modes have the lowest damping. The results of the eigenvalue analysis agree well with those of the nonlinear work analysis and the time domain simulations.
\end{abstract}

\section{Introduction}

In idling mode, the angle of attack (AOA) experienced by the blades significantly varies over one revolution under the combined effects of inflow turbulence, flow inclination and nacelle tilt and yaw. The variation in AOAs remains substantial even in small yaw misalignments within the range of $\pm 15^{\circ}$. It is noted that yaw errors in this range are considered to be within normal idling conditions by wind turbine manufacturers. In moderate yaw angles, the variations in AOA can be such that the rotor enters stall at both positive and negative AOAs, and thereby stall-induced vibrations are likely to occur. In the past, a lot of research effort has been directed to the analysis of stall-induced vibrations (SIVs) in normal operation (Petersen et al., 1998; Hansen, 2003, 2007; Riziotis et al., 2004); however, very little research has been done on parked or idling rotors.
Aeroelastic analysis of parked or idling rotors mostly relies on blade element aerodynamic models. Blade element momentum (BEM) models comply with industry needs for fast aerodynamic tools capable of performing certification simulations. In the context of blade element models, Politis et al. (2009) investigated the stability characteristics of an isolated parked blade at various inflow angles using an eigenvalue approach and considering steady-state but also unsteady aerodynamics. That paper focused on stall-induced instabilities. It was shown that such instabilities can take place at inflow angles that slightly exceed $C_{L \max } \mathrm{AOA}$ but also in the vicinity of $\pm 90^{\circ}$ AOA. In a similar context, Skrzypiński and Gaunaa (2015) investigated the stability of an elastically mounted 2-D section of the parked blade using engineering aerodynamic models. They investigated the effect of imposing temporal lag on the steady-state aerodynamic loads at very high AOA by using indicial functions. Despite the fact that the choice of the lag response was not based on mea- 
sured information and a rather intuitive tuning of the model parameters was performed, it was shown that in all cases an increase in the damping of the low damped edgewise modes is finally obtained even for very small delay values. The authors also investigated the effect of various structural parameters on the damping characteristics of a parked blade. Very recently, Pirrung et al. (2016) proposed a new hybrid model for the analysis of parked or idling rotors. The model is based on the coupling of a trailed vortex near-wake model with a momentum-type far-wake model. The authors only presented time domain aerodynamic analysis results. However, the formulated model is linearizable and can therefore be used for eigenvalue aeroelastic stability analyses.

Although CFD models are still computationally expensive, especially in view of performing extensive time domain certification simulations, some work has been done at the level of advanced aerodynamic models. Skrzypiński et al. (2014a) investigated stall-induced vibrations using 2-D RANS and 3-D DES aerodynamic simulations for a typical elastically mounted blade section in combined flap-edge motion. The 3-D simulations considered an extruded section and periodic spanwise flow conditions. The analysis focused on AOA that slightly exceed $C_{L \max }$. It was concluded that the differences in the predicted post-stall characteristics and the stability limits between 2-D and 3-D CFD analysis are significant. Skrzypiński et al. (2014b) also investigated vortex shedding phenomena occurring at very high AOA in the vicinity of $90^{\circ}$ using CFD models. Also in this work, a typical elastically mounted blade section was considered. AOA of about $90^{\circ}$ can be encountered by a wind turbine blade during the installation phase or in the case of a yaw system failure. Under such flow conditions vortex shedding phenomena can be combined with negative slopes of the lift curve that favor combined stall- and vortex-induced vibrations (VIVs). Typical section analysis showed that lock-on at the vortex shedding frequency is likely to occur depending on the amplitude of the lead-lag motion undergone by the section. Finally, Heinz et al. (2016) and Skrzypinski et al. (2016) analyzed the full blade configuration at $90^{\circ}$ AOA using a DES aerodynamic model coupled to a nonlinear beam model of the blade. The analyses showed that at certain azimuth positions of the parked blade when the inflow has a significant velocity component along the blade axis, spanwise-correlated vortex shedding over large parts of the blade can be triggered, which eventually leads to excessive VIV.

Vortex shedding phenomena and VIVs have also been investigated by Zou et al. (2015) with vortex modeling. Massive flow separation over the blade surface was simulated in the context of the "double wake" concept. The "double wake" concept is based on the modeling principle that the flow separation bubble can be simulated by two vortex sheets, one originating from the trailing edge of the blade and the other from the position at which the separation of the flow takes place. The work focused on a typical elastically mounted blade section at high AOA and showed that VIV and lock-on phenomena are likely to occur at lower wind speeds, while at higher wind speeds edgewise instabilities are mainly driven by the negative slope of the lift curve at AOA in the vicinity of $90^{\circ}$. An important result of this work, which confirms the findings of Skrzypiński and Gaunaa (2015), is that the unsteady character of the flow (temporal lag) increases the damping of the low damped edgewise modes at very high AOA. This indicates that a steady-state analysis is expected to be conservative.

In the present paper, the stability behavior of the conceptual $10 \mathrm{MW}$ reference wind turbine (three-bladed, pitch-regulated, variable-speed turbine with diameter $D=$ $178.3 \mathrm{~m}$ ) designed by DTU Wind Energy (Bak et al., 2013) is assessed in slow idling operation using the linear eigenvalue stability tool GAST_lin (Riziotis et al., 2004). The objectives of the work are to first identify idling situations at moderate yaw misalignment angles that favor stall-induced vibrations and subsequently predict which of the turbine modes exhibit the lowest damping characteristics. The analysis is confined to yaw angles within the range $\left[-60^{\circ},+60^{\circ}\right]$. This is the absolute upper limit up to which engineering dynamic stall models can be trusted. Outside this range, deep stall conditions are encountered that cannot be properly addressed by engineering aerodynamic models. This is because engineering models lack the appropriate tuning in such deep stall conditions. Furthermore, in deep stall, vortex shedding phenomena take place that lead to additional periodic excitation of the rotor not included in the present engineering modeling framework.

The eigenvalue analysis results are compared against the results of nonlinear time domain analysis and the results of aerodynamic work computations from forced harmonic oscillation simulations in which the imposed motion follows the shape and frequency of the turbine modes. This is done in an attempt to demonstrate that fast linear eigenvalue stability tools that have been widely employed by the industry for damping characterization in normal operation conditions can also be trusted for predicting damping in idling operation conditions.

The inflow conditions that favor stall-induced instabilities are identified through turbulent wind nonlinear time domain aeroelastic simulations at yaw angles in the range $\left[-60^{\circ}\right.$, $+60^{\circ}$ ] (moderate to high yaw angles) using the hGAST aeroelastic modeling platform (Manolas et al., 2015). As already mentioned, the above range of yaw angles defines the validated envelope for engineering dynamic stall models. Rotor azimuth positions and corresponding sectional AOAs at which instabilities are favored are assessed. Moreover, the average rotor speeds for the different yaw angles are recorded in order to be used as input to the linear eigenvalue stability tool.

Based on the findings of the time domain analysis, eigenvalue stability analysis is performed focusing on the conditions for which maximum edgewise loads are obtained with hGAST code. Stability analysis in the case of yaw misalign- 
ment requires the application of Floquet theory (Skjoldan and Hansen, 2009; Bottasso and Cacciola, 2015). This is because in yawed flows, periodicity on aerodynamic loads introduced as a result of non-axisymmetric inflow conditions cannot be eliminated through the application of the Coleman multiblade transformation. In addition to the already high cost of Floquet analysis, in idling conditions the rotational speed attains very low values that become at least 10 times smaller than the lowest natural frequency of the turbine, which further increases the cost to a prohibitive level for the analysis intended here. As an alternative, standard eigenvalue analysis could instead be performed on the Coleman-transformed system for coefficients "averaged" over one revolution. However, if this could be done, it would only be in cases of "moderate" periodicity. In the authors' opinion, extending this approach to cases of $30^{\circ}$ or even $60^{\circ}$ yaw angles would definitely be questionable. On the other hand, by noting that at very small rotational speeds ( $\sim 1 \mathrm{RPM})$ low-order harmonics (up to $6 \mathrm{p}$ ) are not expected to interact strongly with the natural frequencies of the turbine, as a second alternative, nonrotating (static) analysis can be performed at different azimuth angles within the sector $\left[0^{\circ}, 120^{\circ}\right]$. The latter approach is followed in the present work. In order to approximate the rotating case as closely as possible, a free-free drivetrain (free rotation boundary condition over the generator side) is simulated, and the idling rotational speed (obtained through time domain simulations) is taken into account in formulating the velocity triangle from the local to the blade section.

Finally, using the aeroelastic mode shapes and frequencies calculated through the eigenvalue analysis as input, aerodynamic work computations are performed. The turbine is set to a prescribed small amplitude harmonic motion following the shape and frequency of the various aeroelastic modes. The work done by the aerodynamic loads acting on the blades as a result of this forced vibration is computed over one oscillation cycle. This work is directly related to the damping of the corresponding mode (Petersen et al., 1998). Nonlinear work computations are compared to eigenvalue analysis results.

The present work is a follow-up to the work presented in Wang et al. (2016). The dynamic stall model used in calculating the aerodynamic loads in deep stall is critical for the assessment of the stability limits of an idling turbine. The innovative contribution of the present work in relation to the previous work presented in Wang et al. (2016) is that emphasis is put on describing in detail the ONERA model employed in hGAST aeroelastic code for the prediction of the aerodynamic loads in dynamic stall (Sect. 3.2). Moreover, a validation of the model in deep stall conditions is presented in the same section. Predictions of the ONERA model are compared to measured data for pitching airfoils in deep stall, well beyond the $C_{L \max } \mathrm{AOA}$, and to results of the state-of-theart Beddoes-Leishman model. Finally, work computations are performed in Sect. 4.3 using both unsteady aerodynamic models and steady-state aerodynamics. In this way, the range of anticipated damping predictions in idling operation is better explored.

The results of the analysis indicate that the asymmetric and symmetric out-of-plane modes exhibit the lowest damping values. Among them, the asymmetric edge horizontal/tilt mode obtains negative damping values at the yaw angle $30^{\circ}$. The results of the eigenvalue analysis agree well with the results of the time domain analysis.

\section{Background on the dynamics and aerodynamic loading of an idling turbine}

In the present section some important features related to the dynamics and aerodynamics of an idling turbine are discussed. This background information is regarded as essential to rendering the stability results presented in the following sections intelligible.

\subsection{Modal characteristics of the idling turbine}

Figure 1 presents the variation in the natural frequencies of the idling reference $10 \mathrm{MW}$ wind turbine versus pitch angle (calculated by hGAST aeroelastic code) as it increases from 0 to $90^{\circ}$ (towards feather). This plot is typically very similar for any three-bladed turbine of similar design philosophy. As expected, the first two tower modes M1 and M2 are not affected at all by the change in the pitch angle. The frequencies of the first asymmetric flap modes M3 and M4 slightly increase, while the frequencies of the first asymmetric edgewise modes M6 and M7 significantly decrease as the pitch angle increases. As a result, asymmetric flapwise and edgewise modes come closer in frequency to each other, despite their initially distinct separation at $0^{\circ}$ pitch angle. Bringing the asymmetric flap and edge modes closer will render the cross-coupling between the corresponding bending directions stronger when the blades are in feather position. The frequency of the first symmetric out-of-plane mode M5 will gradually increase and finally at high pitch angles will exceed the frequency of the asymmetric edgewise modes M6 and M7. The frequency of the first symmetric in-plane (drivetrain) mode M8 decreases with the pitch angle; as the pitch tends to $90^{\circ}$, the frequency of M8 tends to coincide with the frequency of the symmetric out-of-plane mode M5. The second asymmetric flapwise modes M9 and M10 exhibit similar behavior to the first asymmetric flapwise modes M3 and M4.

As the blade pitches to feather, the asymmetric flapwise modes switch from out-of-plane to in-plane. Inversely, the asymmetric edgewise modes switch from in-plane to outof-plane. So, as the blade pitch changes from 0 to $90^{\circ}$, the first asymmetric edgewise (in-plane) vertical mode M6 turns into a first asymmetric edgewise (out-of-pane) yawing mode. The first asymmetric edgewise (in-plane) horizontal mode M7 turns into a first asymmetric edgewise (out-of-pane) tilting mode. This transformation of the asymmetric edgewise modes is depicted in Fig. 2a and b. Inversely, the first asym- 


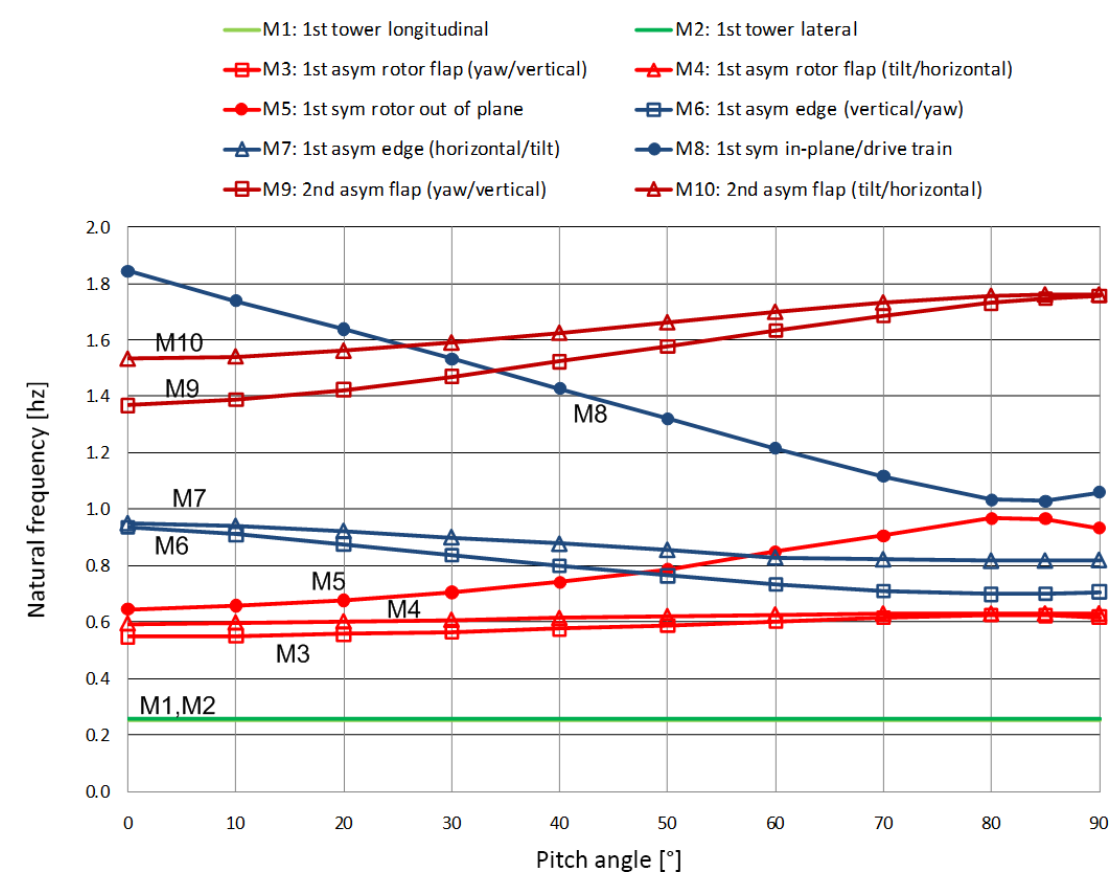

Figure 1. Natural frequencies of the $10 \mathrm{MW}$ reference wind turbine versus pitch from 0 to $90^{\circ}$ (towards feather).
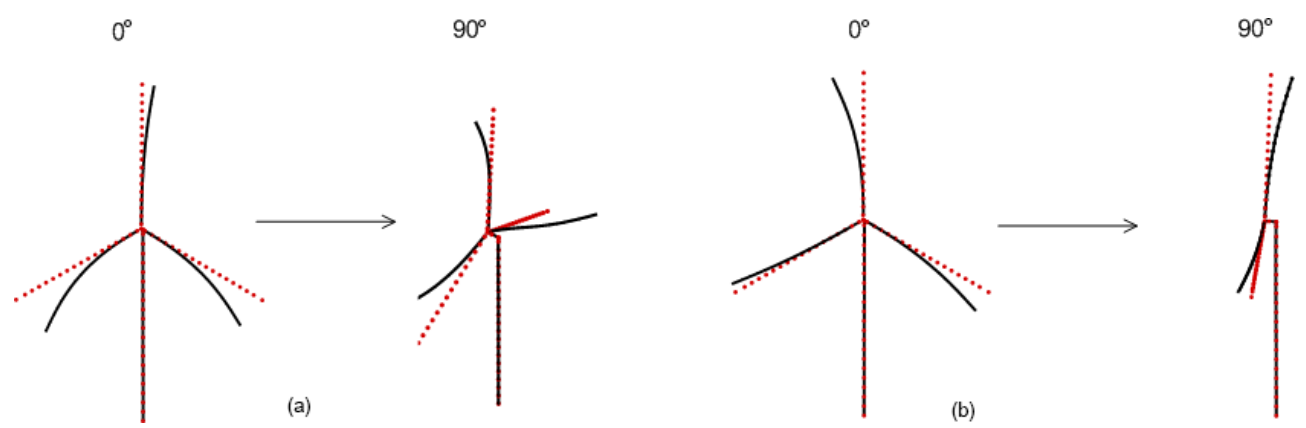

Figure 2. Mode shape alternation from 0 to $90^{\circ}$ pitch for asymmetric edgewise modes (a) M6 and (b) M7. The red symbols indicate an undeformed state, and the black lines indicate a deformed state.

metric flapwise (out-of-plane) yawing mode M3 turns into a first asymmetric flapwise (in-plane) vertical mode, and the first asymmetric flapwise (out-of-plane) tilting mode M4 turns into a first asymmetric flapwise (in-plane) horizontal mode. Similar transformations are obtained in the second flapwise modes M9 and M10.

On the other hand, the symmetric modes seem to retain their original character. As shown in Fig. 3 the out-of-plane (flapwise) collective mode (M5) remains a collective out-ofplane (edgewise) mode as the pitch changes from 0 to $90^{\circ}$. At $0^{\circ}$ pitch, the coupling with the in-plane direction in M5 is negligible. At $90^{\circ}$, as the frequency of M5 gets close to the frequency of the collective in-plane M8 mode, a coupling with the in-plane direction is established.

Also, as shown in Fig. 4, the in-plane (edgewise) collective mode M8 remains a collective in-plane (flapwise) mode as the pitch changes from 0 to $90^{\circ}$. Originally at $0^{\circ}$ pitch, a coupling with the second flapwise modes is clearly noted in the shape of the mode driven by the fact that the collective in-plane mode is close to the second flapwise asymmetric modes. At $90^{\circ}$ pitch, as the frequency of the mode decreases and approaches the frequency of the first collective out-ofplane mode, a coupling with this mode is activated.

\subsection{Aerodynamic characteristics of the idling rotor}

As already discussed, the AOAs experienced by the blades of an idling rotor vary significantly over the revolution. The velocity triangle of an idling blade section is illustrated in Fig. 5 for the blade azimuth positions $0,90,180$ and $270^{\circ}$. It is seen that as a result of yaw error, the AOA reaches a minimum and maximum at $0^{\circ}$ and $180^{\circ}$ azimuth, respectively (whether the AOA will be positive or negative depends on the direction 

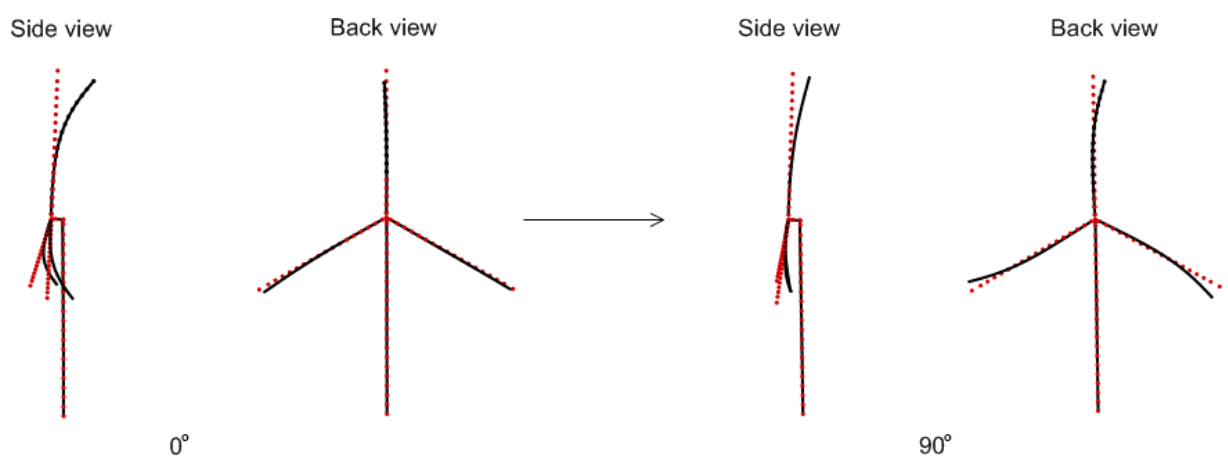

Figure 3. Mode shape alternation from 0 to $90^{\circ}$ pitch for symmetric out-of-plane mode M5. The red symbols indicate an undeformed state, and the black lines indicate a deformed state.

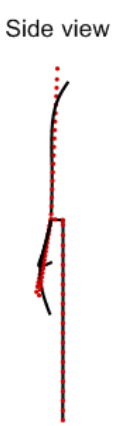

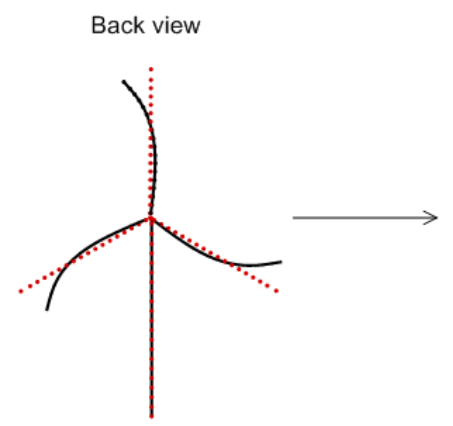

$0^{\circ}$

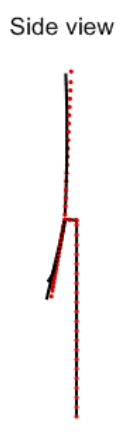

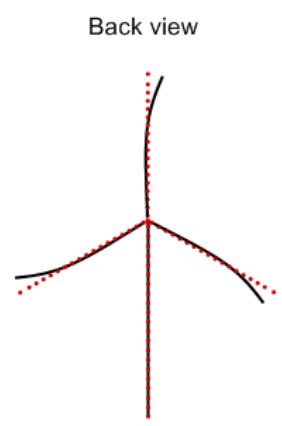

$90^{\circ}$

Figure 4. Mode shape alternation from 0 to $90^{\circ}$ pitch for symmetric in-plane mode M8. The red symbols indicate an undeformed state, and the black lines indicate a deformed state.

of the incoming flow, positive or negative yaw). As a result of the tilt and inflow inclination angles, the AOA attains a minimum at $90^{\circ}$ and a maximum at $270^{\circ}$. An example of the AOA variation in the $75 \%$ section of the reference $10 \mathrm{MW}$ is shown in Fig. 6a for a yaw angle of $15^{\circ}$ and a tilt angle of $5^{\circ}$. The mean wind speed and the pitch setting are taken as $42.5 \mathrm{~m} \mathrm{~s}^{-1}$ and $90^{\circ}$, respectively; the blade section is considered to have zero twist and the linear speed component due to the idling rotation of the blade is not taken into account (assuming an almost zero idling speed). It is seen that as the yaw angle increases, the range of variation in the AOA equally increases. The range of variation in the $\mathrm{AOA}$ is equal to the yaw error angle. Moreover, a superposition of inflow turbulence further increases the range of the AOA variation. If the yaw error is combined with the tilt of the rotor (or the inclination of the mean inflow), the range of variation in the AOA increases further. The azimuth angle for which maximum (positive or negative) AOA is obtained is shifted away from 0 and $180^{\circ}$ given the $90^{\circ}$ phase difference in the two effects. Beyond a certain yaw angle, the blade will definitely enter stall in both the positive and negative AOA regime.

As the pitch of the blade increases towards feather, the local AOAs "seen" by the blade are equally shifted to lower values and therefore idling speed decreases. On the other hand, a reduction in the idling speed leads to higher AOAs along the blade span. An example of the idling speed effect on the AOAs in the $75 \%$ section is shown in Fig. 6b. For an inflow velocity of $42.5 \mathrm{~m} \mathrm{~s}^{-1}$, an almost $10^{\circ}$ increase in the AOAs is noted as the idling speed decreases from 1 RPM to almost 0 RPM. So, the pitch angle of the blade and the rotor idling speed are interrelated but competing parameters as concerns the mean level of the AOA variation.

Whether stall-induced vibrations will appear on an idling rotor depends strongly on the post-stall characteristics of the airfoil sections forming the blade. Higher negative slopes of the $C_{L}$ curve and a deeper drop of the $C_{L}$ in the post-stall region deteriorates stability characteristics. As already mentioned, for moderate to high yaw angles the idling blade enters stall at both positive and negative AOAs. The post-stall characteristics of cambered sections differ significantly between positive and negative AOA. Usually, airfoil sections exhibit smoother post-stall behavior at negative AOAs compared to that at positive ones (there are of course exceptions). This implies that the selection of the appropriate pitch setting and thereby the appropriate idling speed could be critical when targeting the avoidance of stall-induced vibrations. Adjustment of the blade pitch may shift the AOAs to a favorable range. 


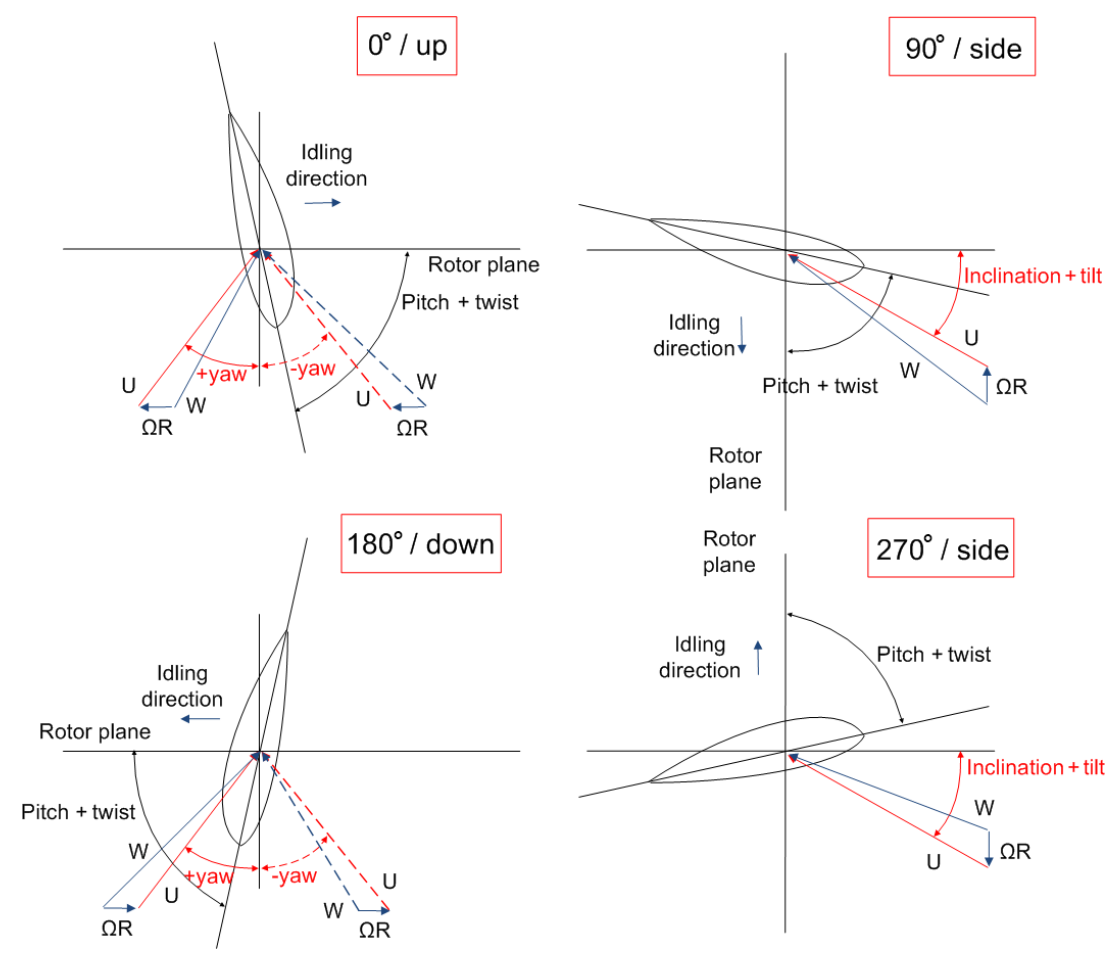

Figure 5. Velocity triangles of an idling blade at different azimuth positions.
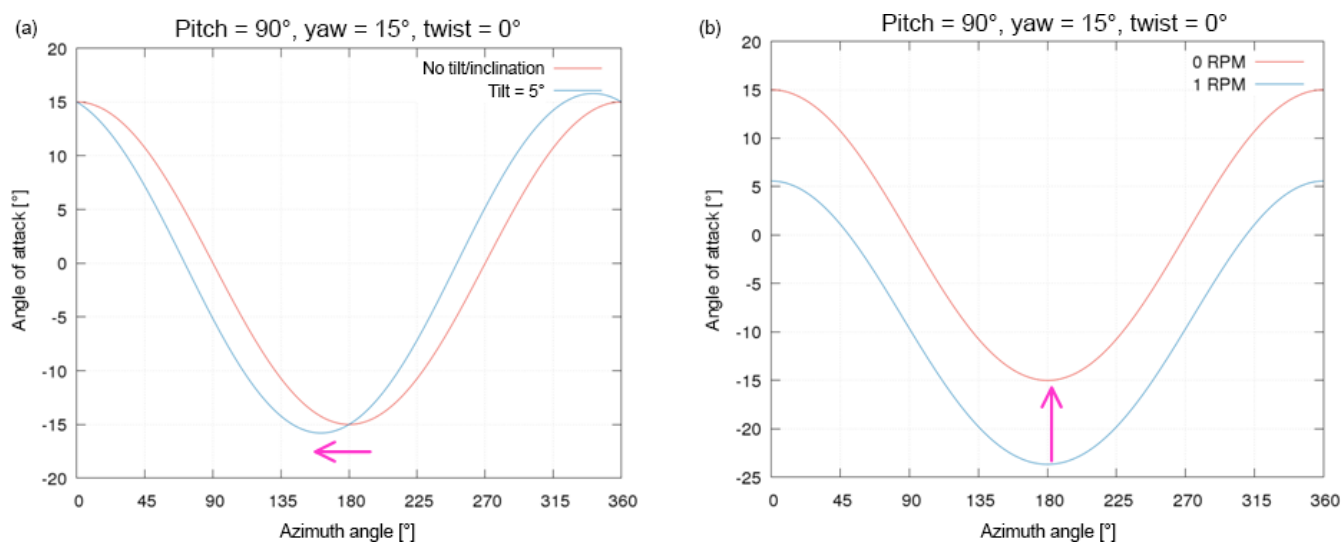

Figure 6. Variation in the angle of attack over one revolution for a wind speed of $42.5 \mathrm{~m} \mathrm{~s}^{-1}$. (a) The effect of the yaw and tilt angle; the tilt angle shifts the curve horizontally, and the rotational speed is almost zero. (b) The effect of rotational speed; the rotational speed shifts the curve vertically.

\section{Description of tools}

\subsection{Description of the aeroelastic tool}

Nonlinear time domain aeroelastic simulations are performed using the NTUA in-house servo-aeroelastic solver hGAST (Manolas et al., 2015). Stability analysis is performed using the eigenvalue stability tool GAST_lin (Riziotis et al., 2004), which is a linearized version of the nonlinear hGAST code. In both the hGAST and GAST_lin solvers, the full wind turbine is considered a multicomponent dynamic system with the main components being the blades, a drivetrain and a tower; all are approximated as Euler-Bernoulli or Timoshenko beam structures. Assembly of the above components into the full system is carried out in the framework of the so-called multibody approach. It consists of considering each component separately from the others but subjected to specific free-body kinematic and loading conditions that are imposed at the connection points of the components.

In the multibody context, a local coordinate system (see Fig. 7a) is assigned to each component with respect to which local elastic displacements are defined. In GAST_lin, the lo- 
(a)

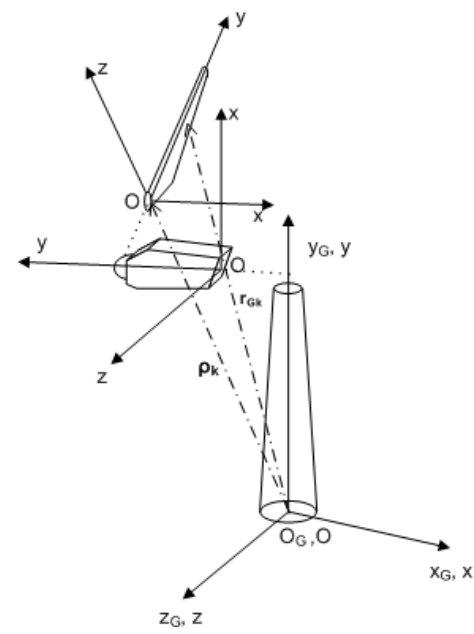

(b)

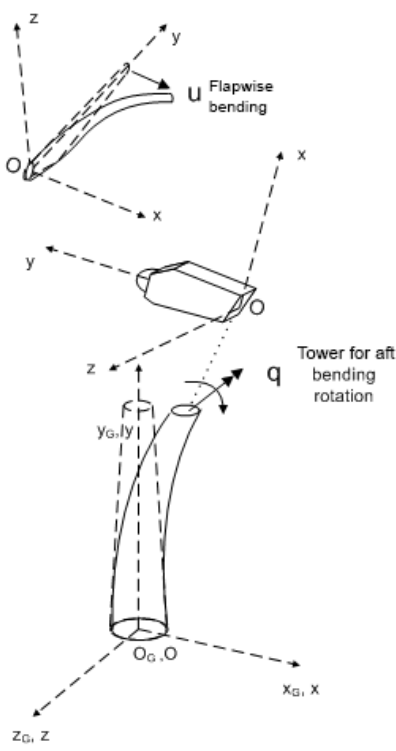

(c)

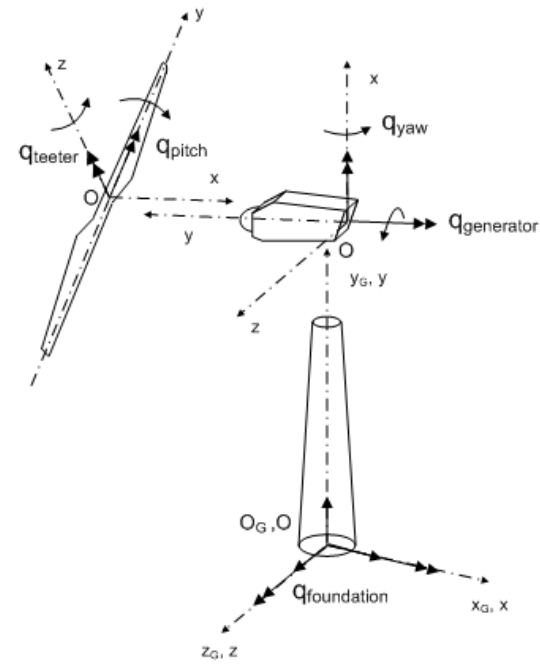

Figure 7. (a) Wind turbine inertial frame and local frame of the various components, (b) realization of multibody kinematics and examples of elastic $q$ DOFs. (c) Realization of multibody kinematics and examples of controlled or free-motion $q$ DOFs.

cal frame of each body is subjected to rigid body and elastic motions communicated by preceding bodies as kinematic conditions imposed at their connection points. Rigid body motions can be either prescribed or controlled, while elastic motions consist of the total deflection of the previous components "transferred" to the current component. For example, the blades are subjected to the elastic translational and rotational motions of the drivetrain and the tower (see Fig. 7b). There are also rigid body motions as the pitch motion and teetering motion of two-bladed rotors (directly imposed on the blades; see Fig. 7c), azimuthal rotation, yaw rotation (indirectly imposed on the blades through the drivetrain and the nacelle; see Fig. 7c), foundation motions or motions of the supporting structure in the case of floating wind turbines (indirectly imposed on the blades through the tower; see Fig. 7c). In addition to the kinematic conditions imposed at the connection points, loading conditions must also be satisfied. In particular, at each connection point, one of the connected bodies contributes to the displacements and rotations of all others, which in turn contributes to their internal (reaction) loads.

The advantage of the above formulation in comparison to other multibody formulations applying the Lagrange multiplier approach is that the resulting dynamic equations of motion can be easily analytically linearized. Thereafter linear eigenvalue stability analysis can be performed with respect to a highly deflected steady or periodic state.

The same multibody formulation is also extendable to the component level, which is actually implemented in hGAST. Highly flexible components, such as the blades, are divided into a number of interconnected sub-bodies, each considered as a single linear beam element or an assembly of linear beam elements. Large deflections and rotations are gradually built and nonlinear dynamics are introduced by imposing on each sub-body the deflections and rotations of preceding sub-bodies as rigid body motions. Dynamic coupling of the sub-bodies is introduced by communicating the reaction loads (three forces and three moments) at the first node of each sub-body to the free node of the previous sub-body as an external load.

Rotor aerodynamics in both codes is simulated using a blade element momentum (BEM) model. In hGAST, an elaborated BEM model is employed that accounts for dynamic inflow, yaw misalignment and dynamic stall effect through the ONERA dynamic stall model (Petot, 1989). In GAST_lin, the frozen wake concept is adopted, while unsteady aerodynamics and dynamic stall effects are again accounted for by means of the ONERA model. In the linearized tool, the unsteady aerodynamic and dynamic stall equations, the corresponding aerodynamic states (circulation parameters of the ONERA model) and the structural equations along with the corresponding DOFs are treated uniformly in one system following the so-called "aeroelastic beam element" concept (Riziotis et al., 2004). It is noted that the effect of gravity is neglected in GAST_lin as it is not expected to affect the stability characteristics of the wind turbine.

In normal operating conditions, stability analysis is performed in the context of multiblade transformation (Coleman and Feingold, 1958). As already discussed in the Introduction, stability analysis of a rotating rotor experiencing yawed inflow would require the application of Floquet theory. This is because periodic coefficients arising from the asymmetry 
in aerodynamic loads cannot be eliminated by means of the Coleman transformation. However, given that idling speeds are usually small ( $\sim 1 \mathrm{RPM})$ and in order to avoid computationally expensive Floquet analysis, stability analysis is performed for a static rotor at different azimuth angles. Azimuth positions in the range $\left[0^{\circ}, 120^{\circ}\right]$ are considered that correspond to one-third of the rotor revolution. Although the rotor is considered static, a free rotation boundary condition is imposed at the generator side in order to approximate idling operation as closely as possible. Also, the average idling rotational speed obtained through the time domain analyses is taken into account in forming velocity triangles local to the blade sections and in calculating the induction parameters.

\subsection{Description of the ONERA model}

As already noted, dynamic stall modeling is essential for the consistent computation of the aerodynamic loads of an idling rotor. In this respect, the extended ONERA model (Petot, 1989) used in the hGAST software and its implementation in the aeroelastic tool are described. Furthermore, some model validation cases for pitching airfoils are presented at the end of the section.

In the extended ONERA model the aerodynamic loads are split into a potential part (indicated by the index 1) and a separated part (indicated by the index 2). The lift and drag forces and the twisting moment of a blade section are given by the expressions

$$
\begin{aligned}
L & =L_{1}+L_{2}=\underbrace{\frac{\rho c}{2}\left(W_{\mathrm{eff}} \Gamma_{1 L}+\frac{s^{L} c}{2} \dot{w}_{0}+\frac{k^{L} c}{2} \dot{w}_{1}\right)}_{L_{1}}+\underbrace{\frac{\rho c}{2} W_{\mathrm{eff}} \Gamma_{2 L}}_{L_{2}} \\
D & =D_{1}+D_{2}=\underbrace{\frac{\rho c}{2}\left(W_{\mathrm{eff}}^{2} C_{D \operatorname{lin}}+\frac{\sigma^{D} c}{2} \dot{w}_{0}\right)}_{D_{1}}+\underbrace{\frac{\rho c}{2} W_{\mathrm{eff}} \Gamma_{2 D}}_{D_{2}} \\
M & =M_{1}+M_{2} \\
& =\underbrace{\frac{\rho c^{2}}{2}\left(W_{\mathrm{eff}}^{2} \cdot C_{M \operatorname{lin}}+\frac{\left(\bar{\sigma}^{M}+d^{M}\right) \cdot c}{2} \dot{w}_{0}+\sigma^{M} W_{\mathrm{eff}} \cdot w_{1}+\frac{s^{M} c}{2} \cdot \dot{w}_{1}\right)}_{M_{1}} \\
& +\underbrace{\frac{\rho c^{2}}{2} W_{\mathrm{eff}} \cdot \Gamma_{2 M}}_{M_{2}},
\end{aligned}
$$

where $\rho$ is the air density, $c$ is the local blade chord, $W_{\text {eff }}$ is the local effective flow velocity at every blade section, $w_{0}$ is the effective flow velocity component normal to the section chord and $w_{1}$ is a rotation velocity parameter due to torsion (both velocity components are explained in Fig. 8). This is given by

$w_{0}=W_{\text {eff }} \sin \alpha_{\text {eff }}$

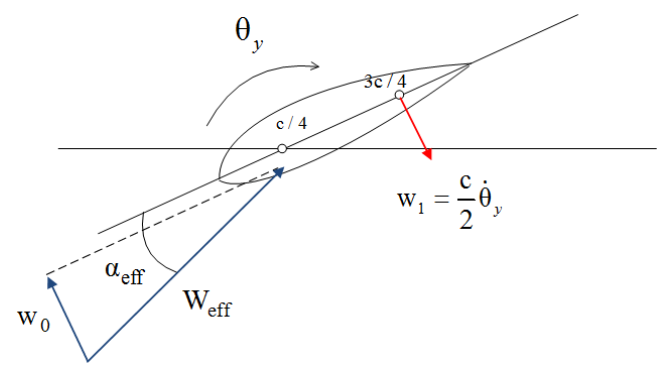

Figure 8. Local velocity components of the ONERA model.

where $\alpha_{\text {eff }}$ is the local flow incidence and $\theta_{y}$ is the local torsion angle of the section (the dot denotes differentiation with respect to time). In Eq. (1), $\Gamma_{1 L}$ and $\Gamma_{2 L}$ are two circulation components (normalized by the semi-chord $c / 2$ ) that define lift force. The first corresponds to attached flow (potential flow lift), while the second provides a necessary correction to account for the effect of flow separation (correction due to stall). Similarly, $\Gamma_{2 D}$ and $\Gamma_{2 M}$ are two "equivalent" circulation components for the calculation of the correction due to flow separation on the drag and the twisting moment, respectively. $C_{D \text { lin }}$ and $C_{M \text { lin }}$ are explained in Fig. 9. Finally, $s^{L}, k^{L}, \sigma^{D}, \bar{\sigma}^{M}, d^{M}, \sigma^{M}$ and $s^{M}$ are the model parameters. Expressions of all model parameters are provided at the end of the section.

It is noted that $L_{1}$ and $M_{1}$ in Eq. (1) are consistent with Theodorsen's model description for a pitching and plunging airfoil section. The first term in the $L_{1}$ equation is the circulatory lift component; the second term is the added mass contribution due to plunging motion (mainly due to flapping motion), and the third term is the added mass contribution due to the section pitching motion (torsion rotation). Similar circulatory and added mass contributions appear in the $M_{1}$ equation.

The two equivalent circulation parameters of the lift force $\Gamma_{1 L}$ and $\Gamma_{2 L}$ are defined through the solution of a first- and second-order differential equation, respectively:

$$
\begin{gathered}
\dot{\Gamma}_{1 L}+\frac{\lambda^{L}}{\tau} \Gamma_{1 L}=\frac{1}{2} \frac{\lambda^{L}}{\tau}\left(\frac{\mathrm{d} C_{L}}{\mathrm{~d} \alpha}\right)_{\operatorname{lin}} W_{\mathrm{eff}} \sin \left[2\left(\alpha_{\mathrm{eff}}-\alpha_{0}\right)\right] \\
+\frac{\lambda^{L}}{\tau} \sigma^{L} w_{1}+\left(\alpha^{L}\left(\frac{\mathrm{d} C_{L}}{\mathrm{~d} \alpha}\right)_{\mathrm{lin}}+d^{L}\right) \dot{w}_{0}+\alpha^{L} \sigma^{L} \dot{w}_{1} \\
\ddot{\Gamma}_{2 L}+\frac{a^{L}}{\tau} \dot{\Gamma}_{2 L}+r^{L} \frac{\Gamma_{2 L}}{\tau^{2}}=-\left[\frac{r^{L}}{\tau^{2}} W_{\mathrm{eff}}\left(\Delta C_{L}\right)+\frac{E^{L}}{\tau} \dot{w}_{0}\right] .
\end{gathered}
$$

The equivalent circulation parameters of the drag force $\Gamma_{2 D}$ and pitching moment $\Gamma_{2 M}$ are defined through the solution to two second-order differential equations (similar in form to the equation for $\Gamma_{2 L}$ ): 

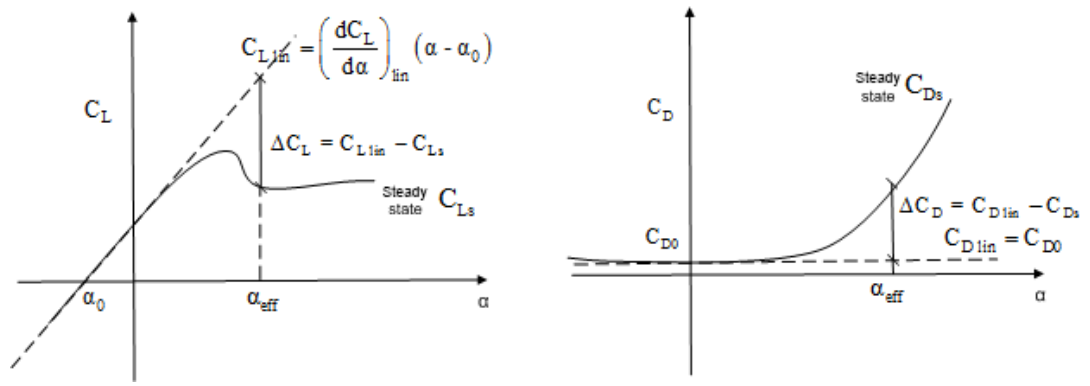

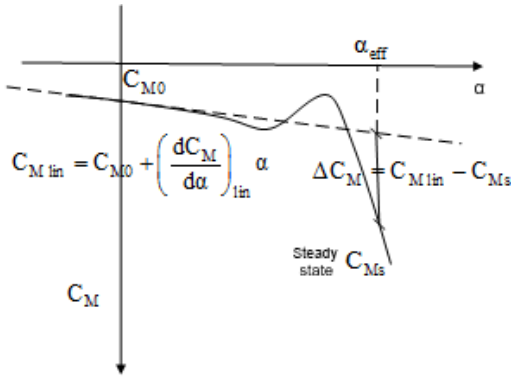

Figure 9. Static polar-dependent parameters in the ONERA model.

$$
\begin{aligned}
& \ddot{\Gamma}_{2 D}+\frac{a^{D}}{\tau} \dot{\Gamma}_{2 D}+r^{D} \frac{\Gamma_{2 D}}{\tau^{2}}=-\left[\frac{r^{D}}{\tau^{2}} W_{\mathrm{eff}}\left(\Delta C_{D}\right)+\frac{E^{D}}{\tau} \dot{w}_{0}\right] \\
& \ddot{\Gamma}_{2 M}+\frac{a^{M}}{\tau} \dot{\Gamma}_{2 M}+r^{M} \frac{\Gamma_{2 M}}{\tau^{2}}=-\left[\frac{r^{M}}{\tau^{2}} W_{\mathrm{eff}}\left(\Delta C_{M}\right)+\frac{E^{M}}{\tau} \dot{w}_{0}\right] .
\end{aligned}
$$

In Eq. (3), $\left(\frac{\mathrm{d} C_{L}}{\mathrm{~d} \alpha}\right)_{\operatorname{lin}}$ is the slope of the linear part of the $C_{L}-\alpha$ curve, and $\left(\Delta C_{L}\right),\left(\Delta C_{D}\right)$ and $\left(\Delta C_{M}\right)$ are differences in the real steady-state viscous lift, drag and moment coefficients from their "linear" counterparts at $\alpha_{\text {eff }}$ (as explained in Fig. 9); $\tau$ is a time parameter given by $\tau=\frac{c}{2 W_{\text {eff }}}$, and $\lambda^{L}, \sigma^{L}, \alpha^{L}, d^{L}, a^{L}, r^{L}, E^{L}, a^{D}, r^{D}, E^{D}, a^{M}, r^{M}$ and $E^{M}$ are the model parameters with $a^{\ell}, r^{\ell}, E^{\ell}=f\left(\Delta C_{L}^{2}\right)$ and $\ell=L, D, M$.

Expressions of all model parameters appearing in Eqs. (1), (3), (4) and (5) are given below as functions of the model constants. Compressibility of the flow is taken into account through the expression of various parameters as functions of the local flow Mach number $M$ defined with respect to $W_{\text {eff }}$ :

$s^{L}=\pi+5 \pi\left[\left(1-M^{2}\right)^{0.285}-1\right]$,

$k^{L}=\frac{\pi}{2}+1.96 \pi\left(\sqrt{1-M^{2}}-1\right)$

$\lambda^{L}=0.17-0.13 M, \quad \alpha^{L}=0.53+0.25\left(\sqrt{1-M^{2}}-1\right)$,

$\sigma^{L}=2 \pi /\left(\sqrt{1-M^{2}}\right), \quad d^{L}=\sigma_{1}^{L}\left|\Delta C_{L}\right|$

$\sigma^{D}=\sigma_{0}^{D} \cdot \alpha_{\text {eff }}+\sigma_{1}^{D}\left|\Delta C_{L}\right|$

$s^{M}=-\frac{3 \pi}{16} \cdot\left[-1.26-1.53 \cdot \tan ^{-1}(15 \cdot(M-0.7))\right]$,

$\sigma^{M}=\sigma_{0}^{M}+\sigma_{1}^{M}\left|\Delta C_{L}\right|$

$\sigma_{0}^{M}+s^{M}=-\frac{\pi}{2} \cdot\left[1+1.4 \cdot M^{2}\right]$,

$\bar{\sigma}^{M}=-\frac{\pi}{4} \cdot\left[1+1.4 \cdot \mathrm{M}^{2}\right]$

$d^{M}=\sigma_{1}^{M} \cdot\left|\Delta C_{L}\right|$

$\sqrt{r^{\ell}}=r_{0}^{\ell}+r_{2}^{\ell} \Delta C_{L}^{2}, \quad a^{\ell}=a_{0}^{\ell}+a_{2}^{\ell} \Delta C_{L}^{2}$,

$E^{\ell}=E_{2}^{\ell} \cdot \Delta C_{L}^{2}, \quad \ell=L, D, M$.

For the constants in the ONERA model, ranges are proposed in Petot (1989). The selected values of the constants depend on the airfoil geometry, and they should be tuned separately for every different airfoil shape. Since we usually lack measured unsteady aerodynamic data for the different blade airfoils (that could be used to tune the constants), fixed values are defined in hGAST within the ranges proposed by Petot, which give reasonable results for most airfoils. They have been down-selected through a tuning process performed based on several unsteady measurements on pitching airfoils with various geometries (Riziotis, 2003; some examples are shown below) and are reported in Table 1.

Next, the results of the ONERA model are presented and compared against wind tunnel measurements for airfoils undergoing pitching motion at high angles of attack. Pitching motion is defined through the equation $a=\alpha_{0}+a_{1} \sin (\omega t)$, where $\alpha_{0}$ is the mean angle, $\alpha_{1}$ is the amplitude and $\omega$ is the angular velocity of the periodic motion. Given the chord length $c$ of the section and the free-stream velocity $U_{\infty}$, the dimensionless reduced frequency parameter $k=\omega c / 2 U_{\infty}$ is defined. Along with the results of the ONERA model, predictions for the state-of-the-art Beddoes-Leishman (BL) model used in several aeroelastic design tools as a baseline dynamic stall model are also presented (implementation by Hansen et al., 2004). In this way, the performance capabilities and limitations of the two models (ONERA and Beddoes-Leishman) are highlighted.

The first set of cases shown in Fig. 10 concerns NACA 63415 airfoil, while the second set shown in Fig. 11 concerns FFA-W3-241 airfoil (this is the airfoil used in the outer part of the DTU reference wind turbine blade). They have both been tested by DTU in the VELUX open jet wind tunnel at a Reynolds number of $R e=1.6 \times 10^{6}$ and a Mach number of $M=0.12$. The test campaign data are reported in Bak et al. (2000) and Fuglsang et al. (1998). Although the above tests are relatively old, they are well suited for the purposes of the present study because they correspond to operation within a stall regime. Usually, dynamic stall tests are run from fully attached to fully separated flow conditions over one period of oscillation. This is not representative of the dynamic stall conditions encountered by an idling blade. Idling blades experience large AOA variations over the period of their rotation (low-frequency variations) but low-amplitude oscillations over periods related to their natural frequencies. 


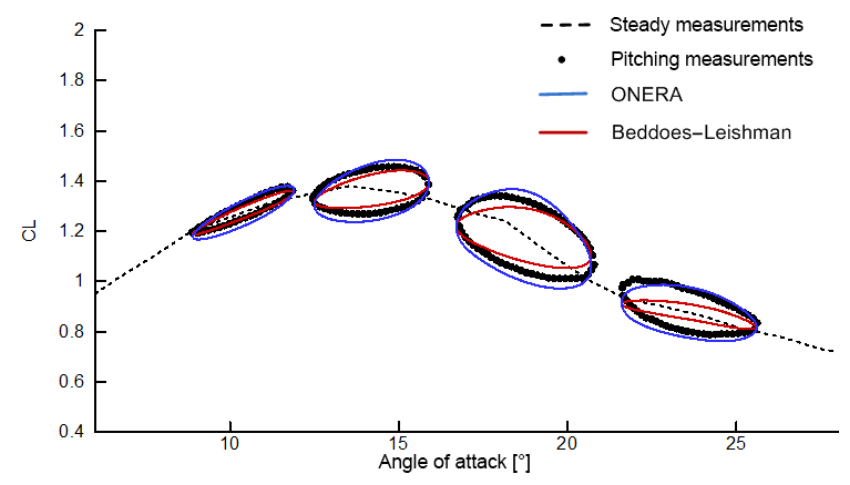

Figure 10. Comparison of engineering dynamic stall models against measurements. Results for a pitching NACA 63415. Mean angles of the four cases $\alpha_{0}=10.4^{0}, 14.2^{0}, 18.7^{0}, 23.6^{0}$; amplitudes of the four cases $\alpha_{1}=1.5^{0}, 1.7^{0}, 2^{0}, 2^{0}$; reduced frequency $k=0.09$ (Bak et al., 2000).

Table 1. ONERA model constants used in hGAST.

\begin{tabular}{lll}
\hline Lift constant & Drag constants & $\begin{array}{l}\text { Pitching moment } \\
\text { constants }\end{array}$ \\
\hline$\sigma_{1}^{L}=0$ & $\sigma_{0}^{D}=0.172, \sigma_{1}^{D}=0$ & $\sigma_{1}^{M}=0$ \\
$r_{0}^{L}=0.18, r_{2}^{L}=0.18$, & $r_{0}^{D}=0.22, r_{2}^{D}=0.2$ & $r_{0}^{M}=0.22, r_{2}^{M}=0.20$ \\
$a_{0}^{L}=0.3, a_{2}^{L}=0.2$, & $a_{0}^{D}=0.25, a_{2}^{D}=0$ & $a_{0}^{M}=0.25, a_{2}^{M}=0.05$ \\
$E_{2}^{L}=-1.5$ & $E_{2}^{D}=-1.15$ & $E_{2}^{M}=1.425$ \\
\hline
\end{tabular}

So, the above tests of low-amplitude oscillations match the conditions encountered by the idling blade and the work simulations performed for the identification of the nonlinear damping characteristics of the rotor. Another point of discussion could be that the above tests correspond to a pitching motion and not to a combined heaving-translation motion as would be expected on an idling blade (a blade undergoing flap-edge motion). However, to the authors' knowledge, no such tests exist, at least not for very high AOA.

The cross-comparison (see Figs. 10 and 11) shows that overall both models perform well, even in deep stall conditions. It is seen that the BL model better predicts the width of the dynamic stall loops in light stall conditions $\left(\alpha_{0}\right.$ angles below $C_{L \max }$ angle). In these light stall cases the ONERA model seems to slightly overpredict the width of the reattachment region, especially for NACA airfoils. For the same airfoil, the ONERA model better predicts the width and the slope of the loops in the deep stall region (beyond $C_{L \max }$ angle). For the FFA-W3-241 airfoil, both models seem unable to correctly predict the slope of the loops, especially at higher mean angles. They both predict a lower slope, which is a clear indication of aerodynamic damping underestimation. The ONERA model predicts a larger and the BL model predicts a smaller width of the dynamic stall loops in deep stall compared to measurements. What is clearly seen in all comparisons is that at very high AOA (highest $\alpha_{0}$ angle), the $\mathrm{BL}$ model considerably underpredicts the width of the loops,

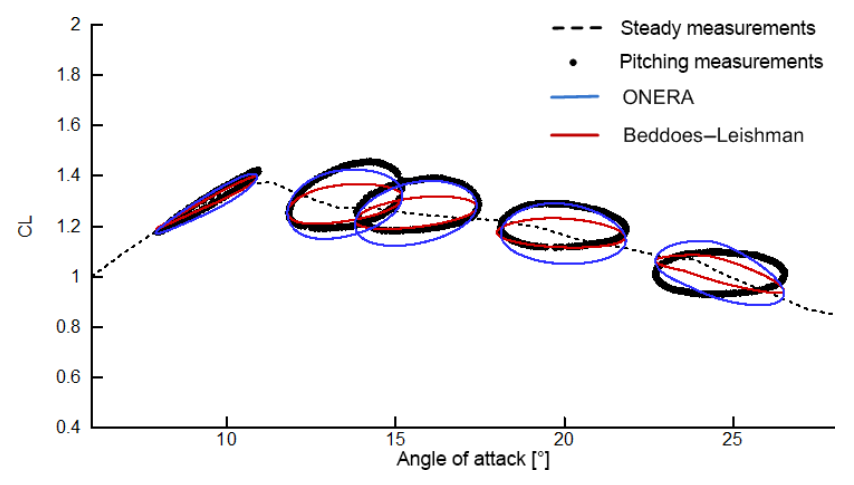

Figure 11. Comparison of engineering dynamic stall models against measurements. Results for a pitching FFA-W3-241. Mean angles of the five cases $\alpha_{0}=9.4^{0}, 13.5^{0}, 15.6^{0}, 19.9^{0}, 24.6^{0}$; amplitudes of the five cases $\alpha_{1}=1.5^{0}, 1.7^{0}, 1.8^{0}, 1.9^{0}, 1.9^{0}$; reduced frequency $k=0.093$ (Fuglsang et al., 1998).

while ONERA consistently provides reasonable results even at these extreme dynamic stall conditions. The above behavior of the BL model is due to its automatic switch to almost steady-state aerodynamics at very high AOA (well beyond $C_{L \max }$ AOA; see Hansen et al., 2004), while the ONERA model is fully deployed at all AOAs.

Despite the reasonable agreement obtained in the presented cases, it cannot be supported that the ONERA model is fully validated in deep stall conditions. Apparently, no dynamic stall model exists that is thoroughly validated in deep stall, and this is mainly because of the lack of relevant measured data for combined heaving-translation motion that could serve its tuning.

By definition, the ONERA model makes no distinction in the treatment of dynamic stall between positive and negative AOAs. The correction of the potential lift is made on the basis of $\Delta C_{L}, \Delta C_{D}$ and $\Delta C_{M}$, which at negative AOA stall are defined in a similar manner as at positive. The model has already been tested in VAWT applications and for low tipspeed ratio values, in which blades go into negative AOA stall on the advancing side and positive AOA stall on the retreating side; predictions for aerodynamic loads have proven to be in good agreement with measurements (see Shi et al., 2014). This is important, since in idling operation the blades experience AOA in both regimes. Thereby, it can be stated that the model functions are equally suitable at negative stall conditions.

For the eigenvalue stability analysis of the rotor and wind turbine system, aerodynamic loads in Eq. (1) and the ONERA differential Eqs. (3), (4) and (5) are linearized analytically and combined with the beam equations in one set that is solved using the FEM method in the context of the so-called "aeroelastic beam element" approach. Additional mass, damping and stiffness matrices are obtained through the linearization of the aerodynamic loads that supplement the structural ones. 


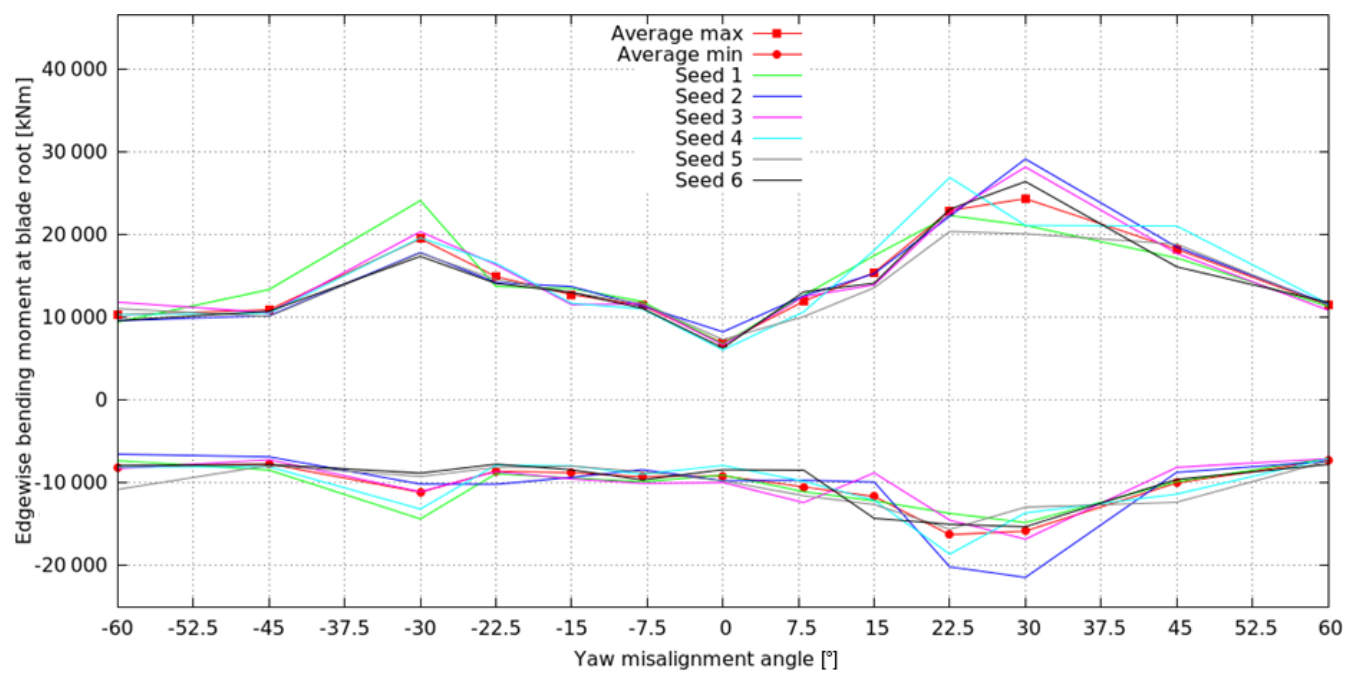

Figure 12. Ultimate blade root edgewise bending moments. Loads from six wind seeds are averaged. Wind speed $42.5 \mathrm{~m} \mathrm{~s}{ }^{-1} ; \mathrm{TI}=11 \%$; yaw angles in the range $\left[-60^{\circ},+60^{\circ}\right]$.

\section{Results and discussion}

\subsection{Time domain analysis results}

Time domain aeroelastic simulations in turbulent inflow are performed for the reference $10 \mathrm{MW}$ wind turbine at a mean wind speed of $42.5 \mathrm{~m} \mathrm{~s}^{-1}$ with a turbulence intensity (TI) of $11 \%$ for various yaw misalignment angles in the range $\left[-60^{\circ},+60^{\circ}\right]$ and a constant pitch angle of $87^{\circ}$. Six $10 \mathrm{~min}$ simulations are performed for every yaw angle corresponding to different realizations of the wind (wind seeds). Figure 12 presents the min-max envelope of the edgewise (outof-plane) bending moments at blade root as functions of the yaw misalignment angle. The load results for all three blades for the different wind seeds and average loads are provided in the plot. It is seen that ultimate loads attain both maximum and minimum value at $+30^{\circ}$ of yaw. When loads are averaged, the minimum is found at $+22.5^{\circ}$ of yaw (very close to the load at $+30^{\circ}$ of yaw), while the maximum is still obtained at a $+30^{\circ}$ yaw angle.

For the yaw angle $30^{\circ}$, a pattern in the edgewise bending moments at the three blade roots is shown in Fig. 13 along with the time series of the azimuth angle and the AOA for a $45 \mathrm{~s}$ duration. The average idling speed of the rotor is about $0.8 \mathrm{RPM}$ at the above conditions (wind speed $42.5 \mathrm{~m} \mathrm{~s}^{-1}$ and yaw $30^{\circ}$ ). Also in Fig. 14, the obtained $C_{L}$ values at $r / R=90 \%$ are collected and plotted with respect to the corresponding AOA. In this plot, the regions of unfavorable lift slope are marked in gray.

At $t=107 \mathrm{~s}$ (marked with a dashed blue line in Fig. 13), mild edgewise vibrations start to grow on blade 3 as a result of increasing AOAs that push the blade into stall. At $t=107 \mathrm{~s}$, the rotor azimuth is $90^{\circ}$ (rotor azimuth is defined as the azimuth angle of the blade in the sector $\left[0^{\circ}, 120^{\circ}\right]$; blade 1 in this case). It is noted that $0^{\circ}$ azimuth corresponds to a blade being at 12:00. The AOA on blade 3 crosses the level of $15^{\circ}$ so stall takes place at positive AOA. Blade 2 also operates in deep stall, experiencing negative AOA in the range $\left[-30^{\circ},-40^{\circ}\right]$. Finally, the flow remains attached on blade 1 . The AOAs experienced by blade 1 remain at about $-10^{\circ}$ for approximately $5 \mathrm{~s}$, so no vibrations are expected for this blade within this time interval.

As time progresses, the situation changes. At $t=116 \mathrm{~s}$ (marked by a red dashed line), mild edgewise vibrations start to grow on blade 1 as a result of increasing AOA that pushes this blade into stall at the negative AOA regime. As indicated in Fig. 13, at $t=116 \mathrm{~s}$ the azimuth angle of the rotor is $20^{\circ}$ and the AOA "seen" by blade 1 grows to about $-30^{\circ}$. As time progresses (time period [116 s, $120 \mathrm{~s}]$ ), blade 1 goes deeper into negative AOA stall (about $-40^{\circ}$ ), while over the same time period, blade 3 stays within deep stall and experiences AOA in the range $\left[20^{\circ}, 30^{\circ}\right]$. This explains why blade 3 exhibits large vibrations over the whole time period [107 s, $120 \mathrm{~s}$ ]. Return to attached flow conditions is only obtained for blade 3 at $t>120 \mathrm{~s}$. In contrast to the other two blades, blade 2 operates in attached flow conditions (AOA $\sim-15^{\circ}$ ).

Looking back in time at the time interval $[80 \mathrm{~s}, 95 \mathrm{~s}]$, it can be seen that blade 1 experiences similar flow conditions to those experienced by blade 3 in the time interval [ $107 \mathrm{~s}$, $115 \mathrm{~s}]$. Blade 2 is in the azimuth range $\left[80^{\circ}, 100^{\circ}\right]$. The only difference with respect to the time period $[107 \mathrm{~s}, 115 \mathrm{~s}]$ is that the rotor speed is lower and therefore blade 1 remains within the stall region longer. High load amplitudes are obtained for blade 1 within this time interval, which decrease when blade 1 moves away from stall (AOA and loads for blade 1 decrease in the time interval [95 s, $105 \mathrm{~s}])$. 

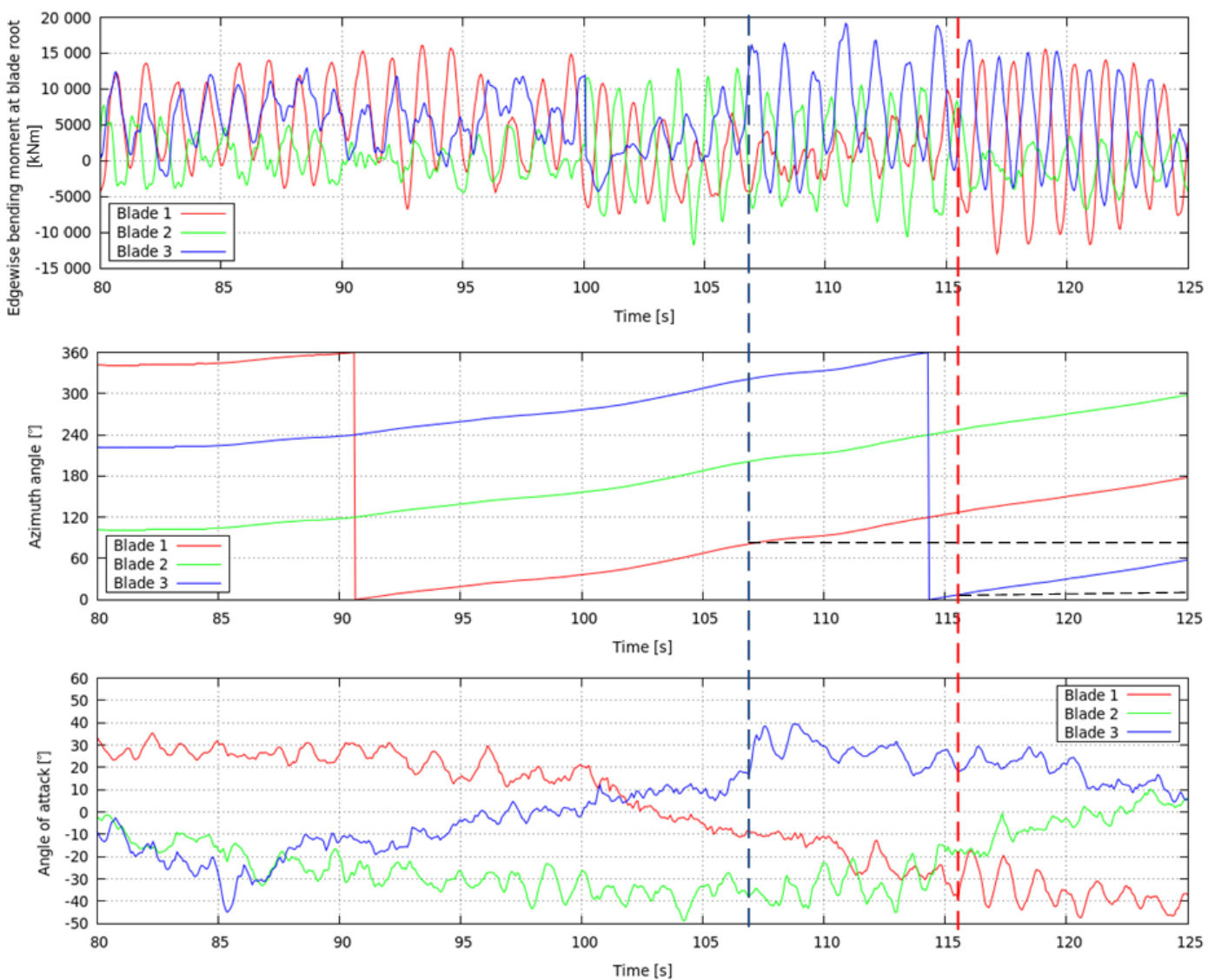

Figure 13. Times series of blade root edgewise bending moment with the blade azimuth angle and angle of attack at $r / R=0.90$ spanwise position. Wind speed $42.5 \mathrm{~m} \mathrm{~s}^{-1}$; TI $11 \%$; yaw angle $30^{\circ}$.

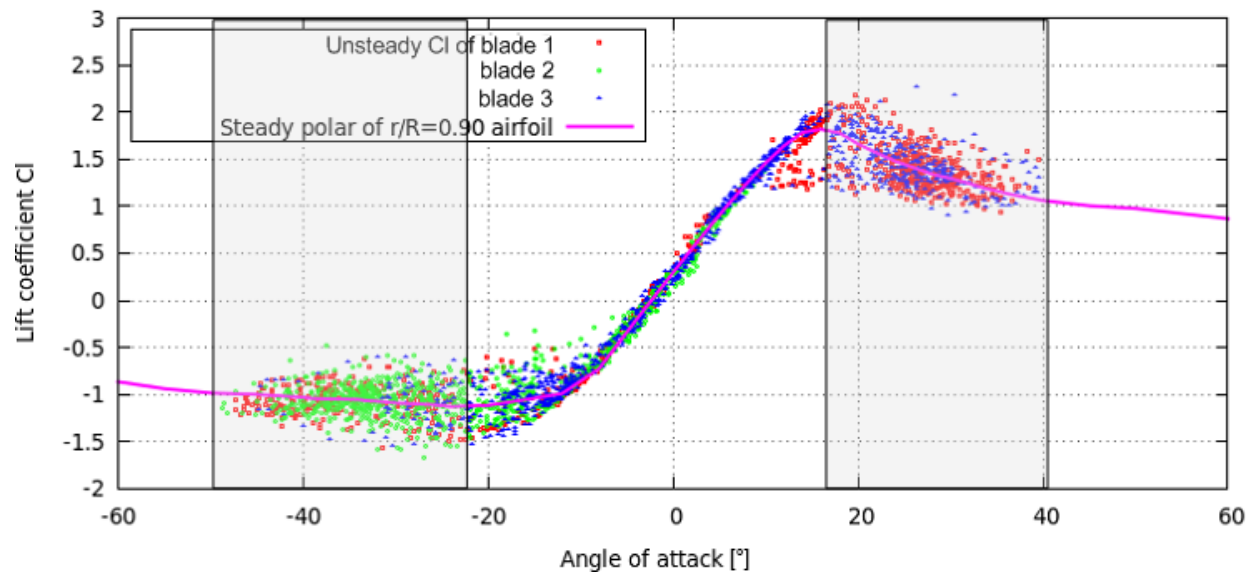

Figure 14. $C_{L}-\mathrm{AOA}$ plot at $r / R=0.90$ spanwise position. Wind speed $42.5 \mathrm{~m} \mathrm{~s}^{-1}$; TI $11 \%$; yaw angle $30^{\circ}$.

\subsection{Eigenvalue analysis results}

In the present section, eigenvalue stability analysis results for the idling rotor are presented at a wind velocity of $42.5 \mathrm{~m} \mathrm{~s}^{-1}$ and for various yaw angles in the range $\left[0^{\circ}, 60^{\circ}\right]$. The average idling speed versus yaw angle in the stability analysis was taken from the time domain simulation results by aver- aging the mean rotor speed of the six realizations performed for every yaw angle. The results of the mean idling speed as a function of the yaw angle are shown in Fig. 15. Emphasis is put on yaw angles for which higher minimum or maximum loads are predicted by nonlinear time domain analysis. A correlation is attempted between the damping and frequency 


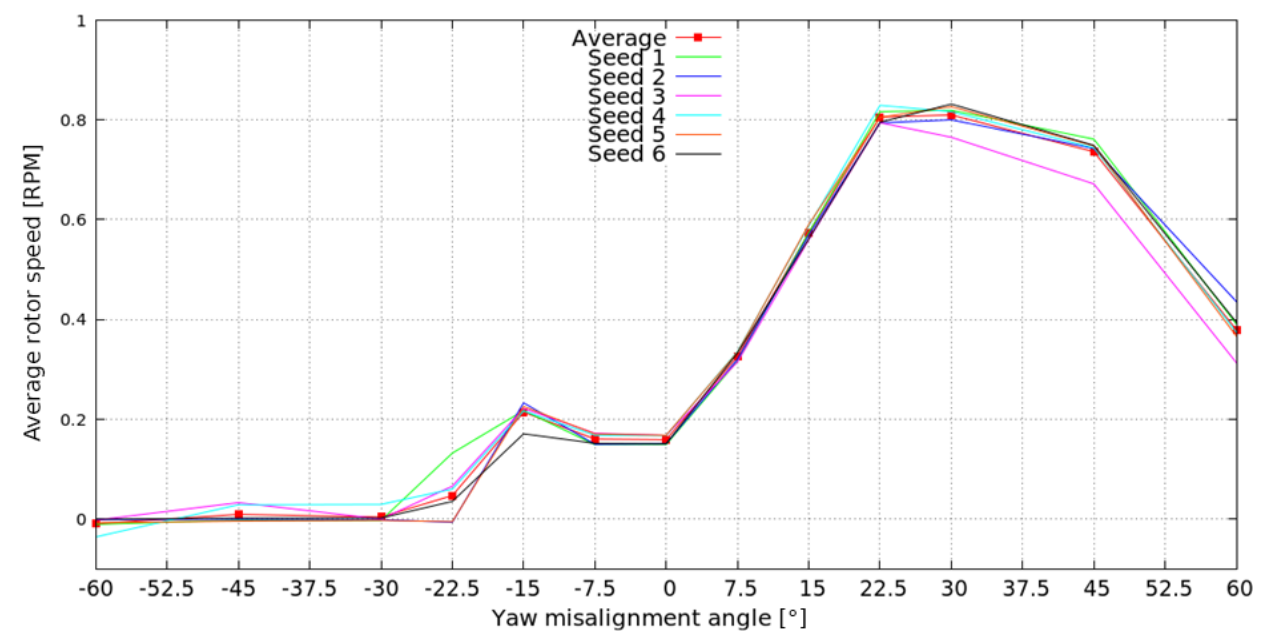

Figure 15. Rotor idling speed versus yaw angle for the pitch angle $87^{\circ}$. Average rotor speed of six different 10 min wind seeds and their average.

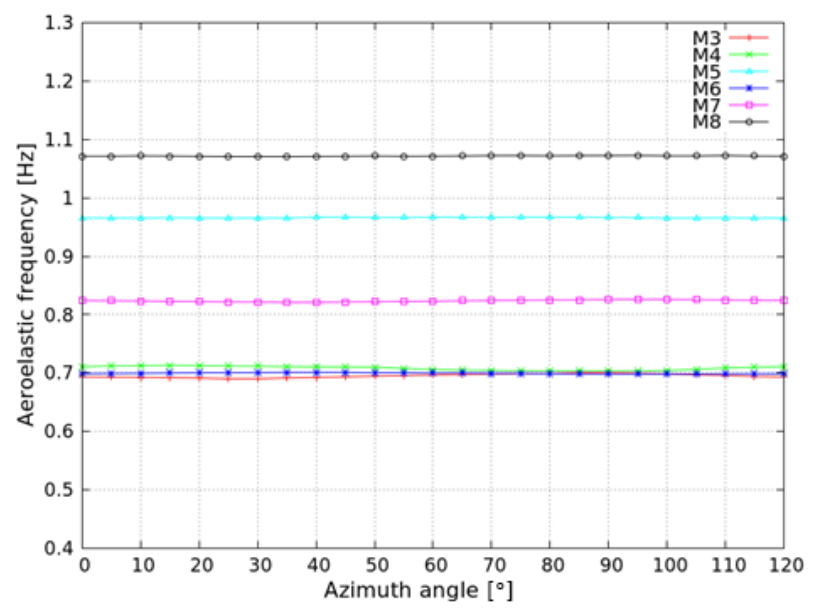

(a)
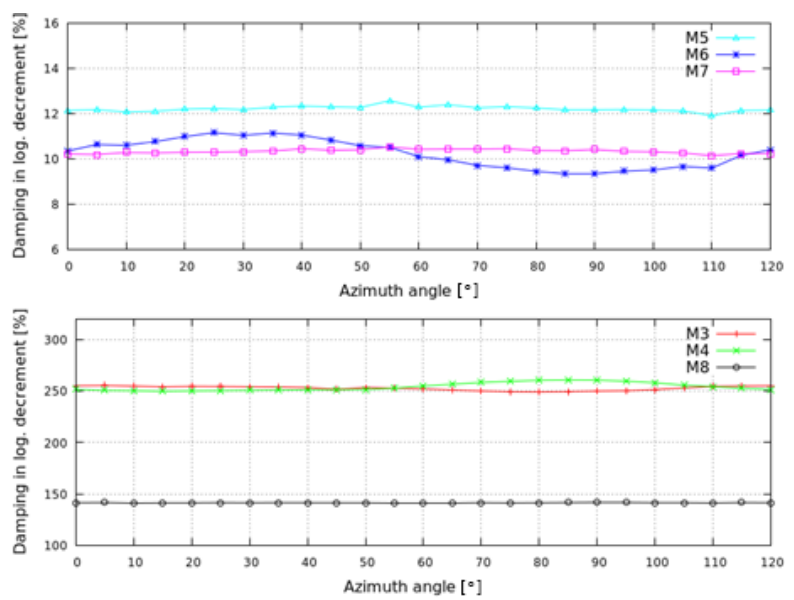

(b)

Figure 16. (a) Modal frequency and (b) modal damping of rotor modes versus azimuth angle at $U=42.5 \mathrm{~m} \mathrm{~s}^{-1}$ and yaw $=0^{\circ}$ (unsteady aerodynamics based on the ONERA model).

predictions obtained through the eigenvalue stability analysis with the results of the time domain analysis. Eigenvalue stability analysis is performed using both steady-state and unsteady (ONERA model) aerodynamics. The feature of the BL model to automatically switch to steady-state aerodynamics at very high AOA necessitated the analysis with steady-state polars. Within the stall region, damping predictions of the steady-state analysis will be the most conservative; by using steady-state polars, the range of anticipated damping predictions can be determined.

The modal frequencies and damping (in logarithmic decrement) of the rotor modes (M3-M8) at $0^{\circ}$ yaw are shown in Fig. 16a and $\mathrm{b}$ as functions of the rotor azimuth angle in the range $\left[0^{\circ}, 120^{\circ}\right]$ (azimuth angle of blade 1). Results are obtained using the ONERA unsteady aerodynamics model. In Fig. 17a and $b$ the PSDs of the flapwise and edgewise bend- ing moments from the earlier time domain simulations are also provided for the same yaw angle.

It is seen that the three out-of-plane modes, M5, M6 and M7, are clearly the lowest damped (Fig. 16b). This is definitely in agreement with the time domain analysis results as shown in the PSD plots of the edgewise bending moment at the root of the three blades (Fig. 17b). The three predominant peaks on the PSD plot indicate the modes that are highly excited. The corresponding frequencies are found at $\sim 0.69$, 0.81 and $0.95 \mathrm{~Hz}$. These frequencies agree well with the values obtained with the stability tool (Fig. 16a). A lower peak appears at $0.25 \mathrm{~Hz}$, which corresponds to the first longitudinal tower bending mode M1. No peak appears in the PSD of the blade root flapwise bending moment (Fig. 17a). This indicates that the aerodynamic damping of the in-plane modes M3, M4 and M8 is high. The same result is consistently pre- 


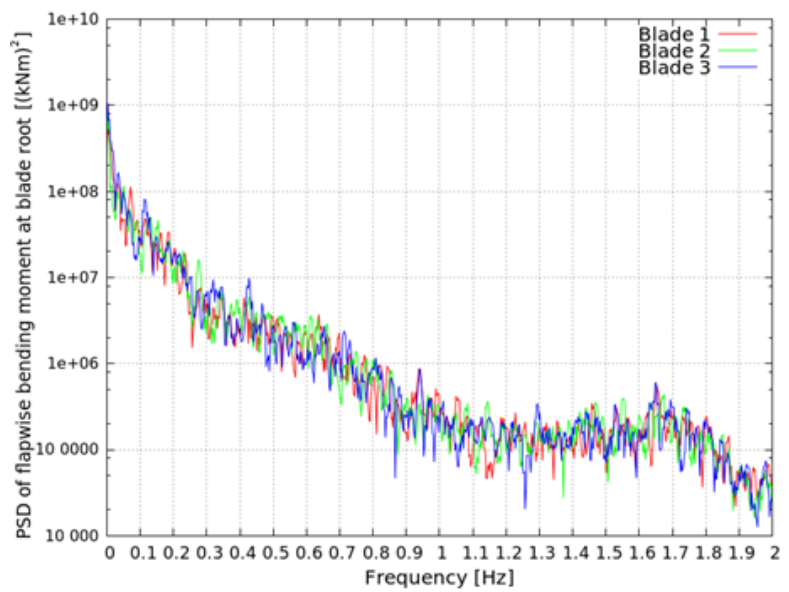

(a)

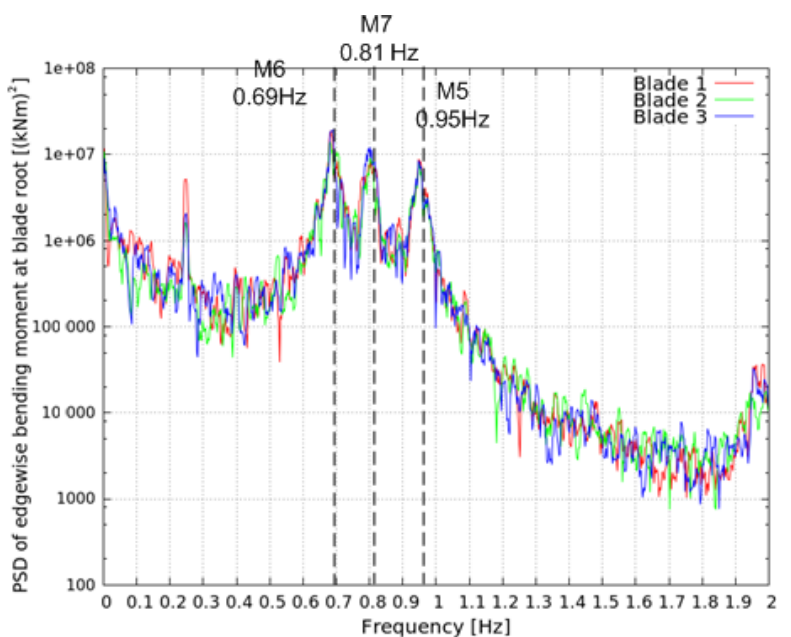

(b)

Figure 17. (a) PSD of the flapwise bending moment at the blade root and (b) PSD of the edgewise bending moment at the blade root; $U=42.5 \mathrm{~m} \mathrm{~s}^{-1}$ and yaw $=0^{\circ}$.

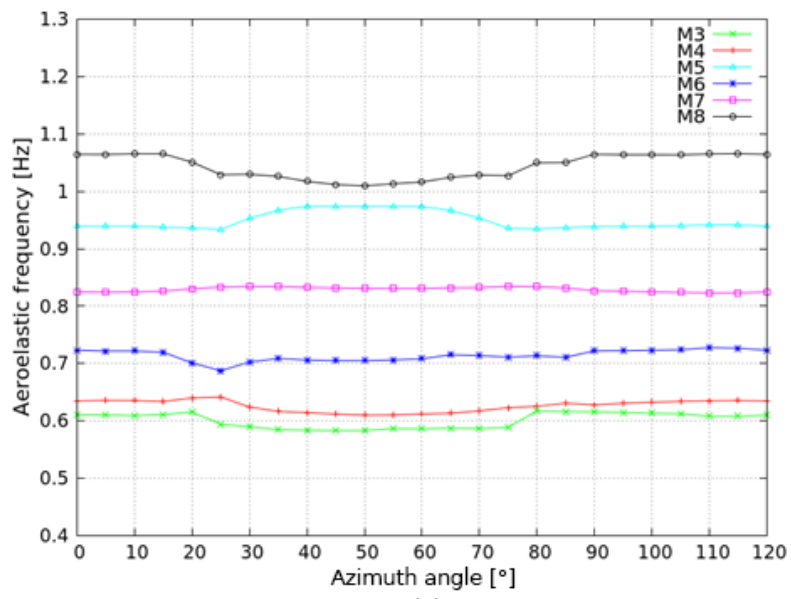

(a)

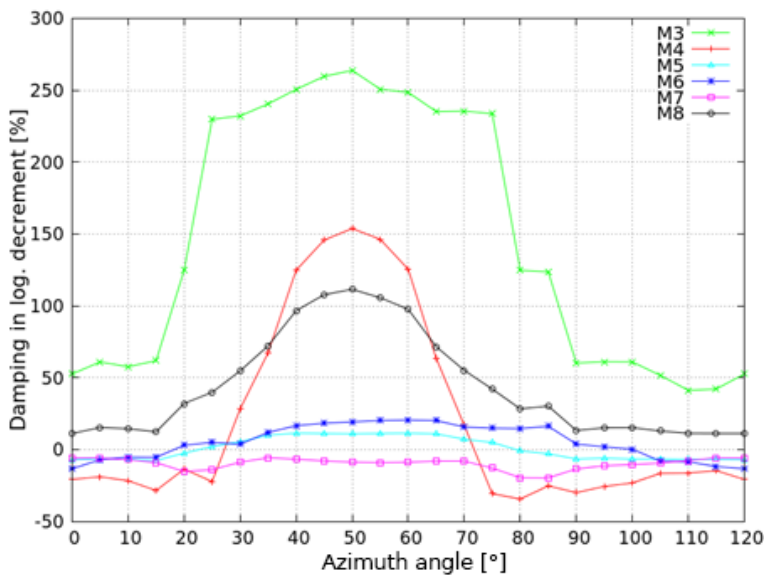

(b)

Figure 18. (a) Modal frequency and (b) modal damping of rotor modes versus azimuth angle at $U=42.5 \mathrm{~m} \mathrm{~s}^{-1}$ and yaw $=30^{\circ}$ (steady-state aerodynamics).

dicted by the stability tool (all values are above $100 \%$ in logarithmic decrement). It is worth noting that both the level of damping and the frequencies are almost independent of the azimuth position in the $0^{\circ}$ yaw case. Eigenvalue analysis using steady-state polars (not shown in the figures) produces similar results to those from the unsteady aerodynamic analysis. The damping of the low damped out-of-plane (edgewise) modes M5, M6 and M7 remains positive at all azimuth angles, while in-plane (flapwise) modes appear to have about $50 \%$ higher damping and $20 \%$ lower frequency. The damping and frequencies are also almost independent of the azimuth angle when steady-state aerodynamics is used.

Figure 18a and $\mathrm{b}$ present frequency and damping results for the yaw angle $30^{\circ}$ using steady-state aerodynamics. The same set of results using unsteady aerodynamics is presented in Fig. 19a and b. Figure 20a and b present the PSDs of the flapwise and edgewise bending moments at the blade root of the three blades as predicted through nonlinear time domain simulations. According to the time domain analysis results in Sect. 4.1, at $30^{\circ}$ of yaw the edgewise loads reach their maximum values. Also, as explained in Sect. 2, for this high yaw angle the AOAs experienced by the rotating blades will significantly vary with the azimuth angle. As a result, modal frequencies and damping also vary over the revolution in contrast to the $0^{\circ}$ yaw case. At very high yaw angles, the AOAs seen by the blades are expected to enter post-stall on both sides of the polar (positive and negative AOAs).

Compared to the $0^{\circ}$ yaw case, lower damping values are obtained for most modes (in-plane and out-of-plane). This indicates that stall-induced vibrations start to develop at the 


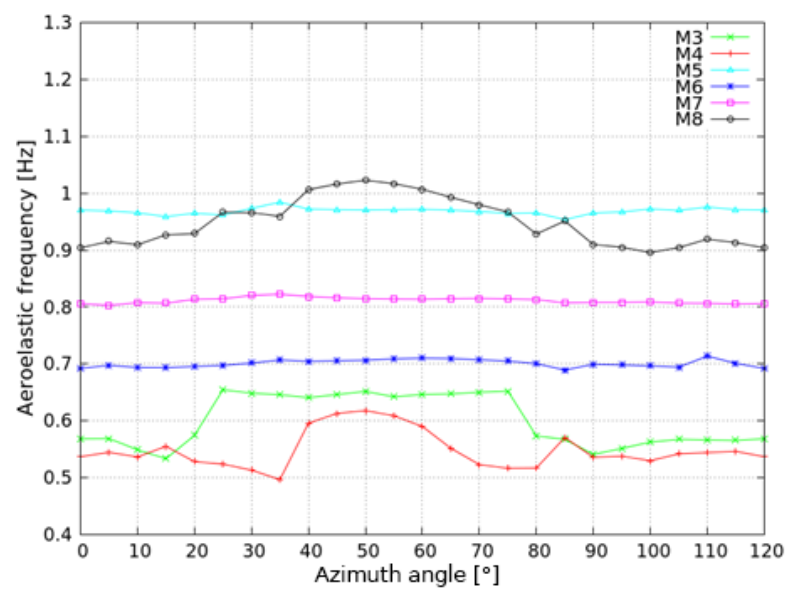

(a)

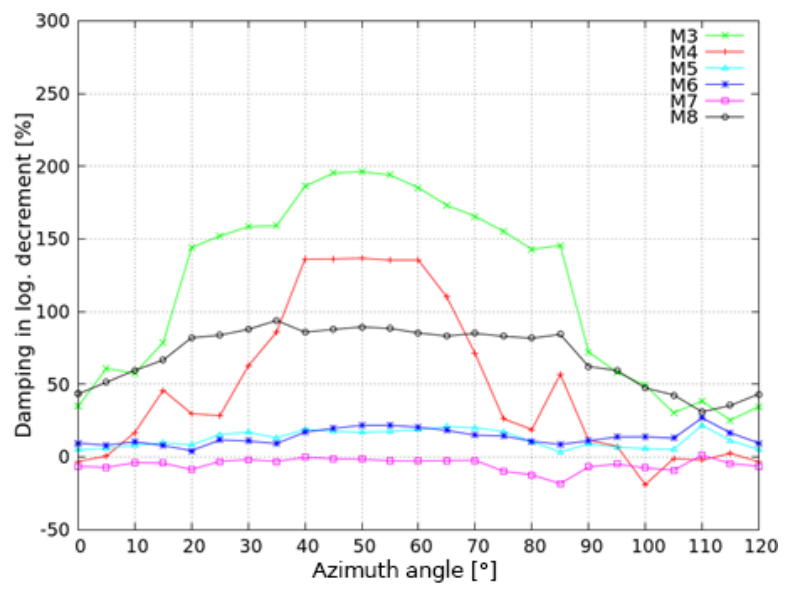

(b)

Figure 19. (a) Modal frequency and (b) modal damping of rotor modes versus azimuth angle at $U=42.5 \mathrm{~m} \mathrm{~s}^{-1}$ and yaw $=30^{\circ}$ (unsteady aerodynamics based on the ONERA model).
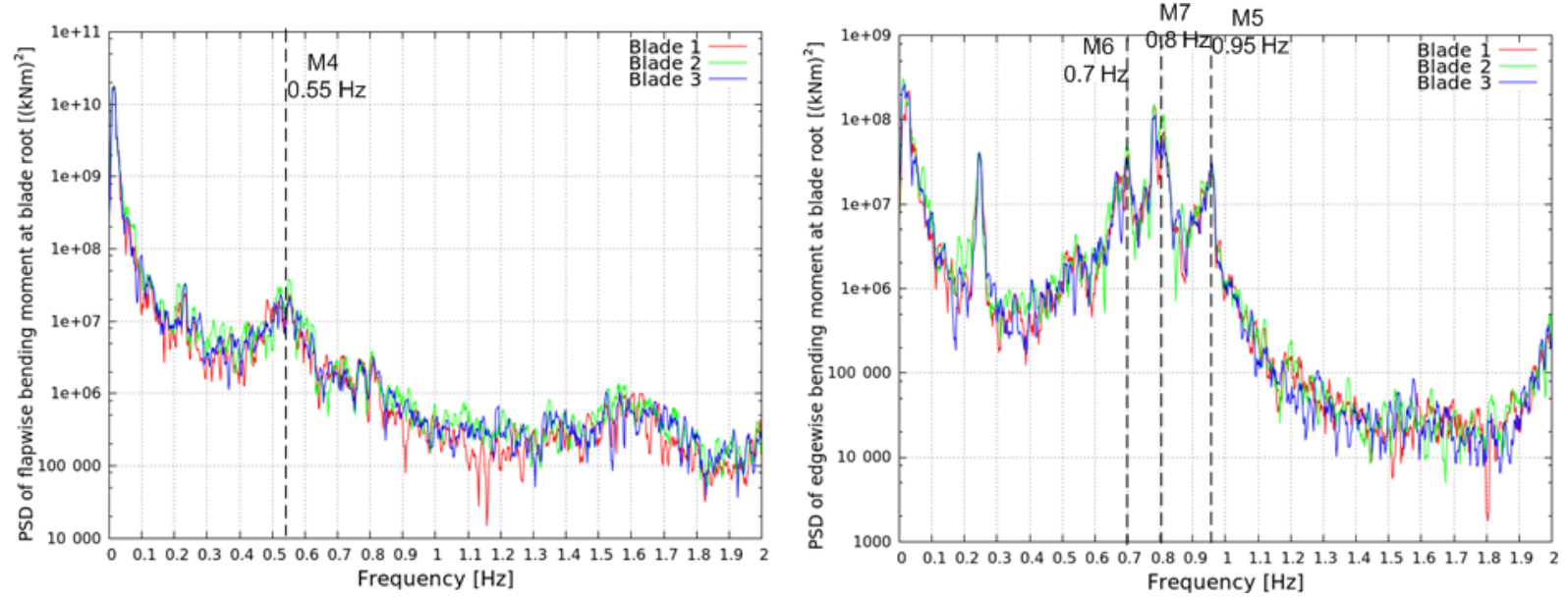

Figure 20. (a) PSD of the flapwise bending moment at the blade root and (b) PSD of the edgewise bending moment at the blade root; $U=42.5 \mathrm{~m} \mathrm{~s}^{-1}$ and yaw $=30^{\circ}$.

yaw angle $30^{\circ}$. The damping of the in-plane modes M3, M4 and M8 significantly drops. The damping of M4 (asymmetric flap tilt/horizontal mode) becomes negative at certain azimuth angles. When steady-state polars are used, the damping of M4 is negative at the azimuth ranges $\left[0^{\circ}, 25^{\circ}\right]$ and $\left[75^{\circ}, 120^{\circ}\right]$. Almost constant, highly negative damping values of about $-25 \%$ are obtained for M4 in the above azimuth ranges. When the ONERA unsteady aerodynamics model is used, the damping becomes locally negative only at the azimuth angles 0 and $100^{\circ}$. Despite the negative damping values of M4, damping remains highly positive (up to $150 \%$ ) over a wide azimuth range $\left[30^{\circ}, 70^{\circ}\right]$. This suggests that the overall damping of the in-plane modes will eventually be positive. On the other hand, modes M5, M6 and M7 still have low or negative damping throughout the entire range of azimuth angles. Especially for M7 (asymmetric edge horizontal/tilt mode), the damping is negative at all azimuth angles for both steady-state and unsteady aerodynamics. Comparing the two aerodynamic models, the damping of M7 is lower at all azimuth angles when steady-state polars are used. For M5 and M6, the damping predicted with unsteady aerodynamics remains low but positive at all azimuth angles, while with steady-state aerodynamics the damping is negative in the azimuth ranges $\left[0^{\circ}, 20^{\circ}\right]$ and $\left[90^{\circ}, 120^{\circ}\right]$.

The stability results that used unsteady aerodynamic modeling are in line with the observations made in the time domain analysis in the previous section. In agreement with the time domain analysis results, higher negative damping values are noted over the azimuth ranges $\left[0^{\circ}, 20^{\circ}\right]$ and $\left[75^{\circ}, 110^{\circ}\right]$ (azimuth ranges in which higher vibrations have been observed in time domain results). Again, four peaks dominate the PSD plot of the edgewise bending moment at $0.25,0.7$, 0.8 and $0.95 \mathrm{~Hz}$ (see Fig. 20b). The first corresponds to the first tower longitudinal bending mode M1, while the other 


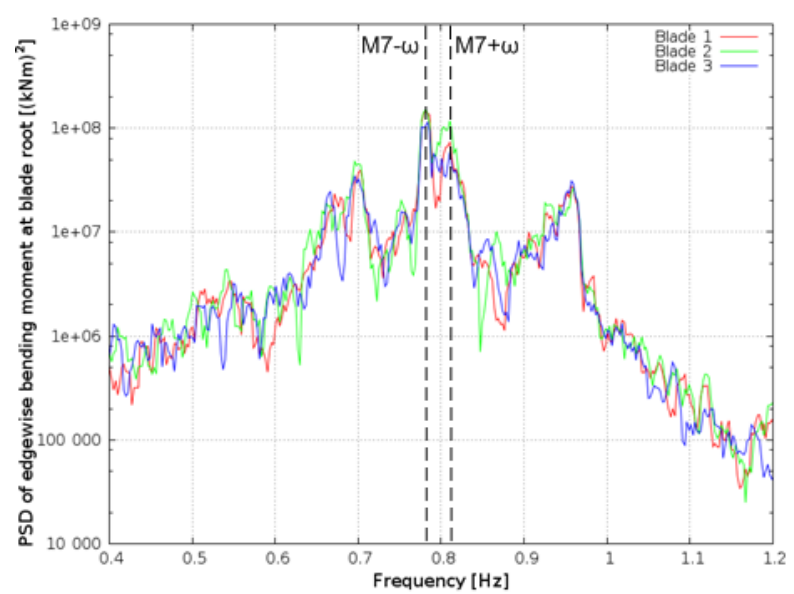

(a)

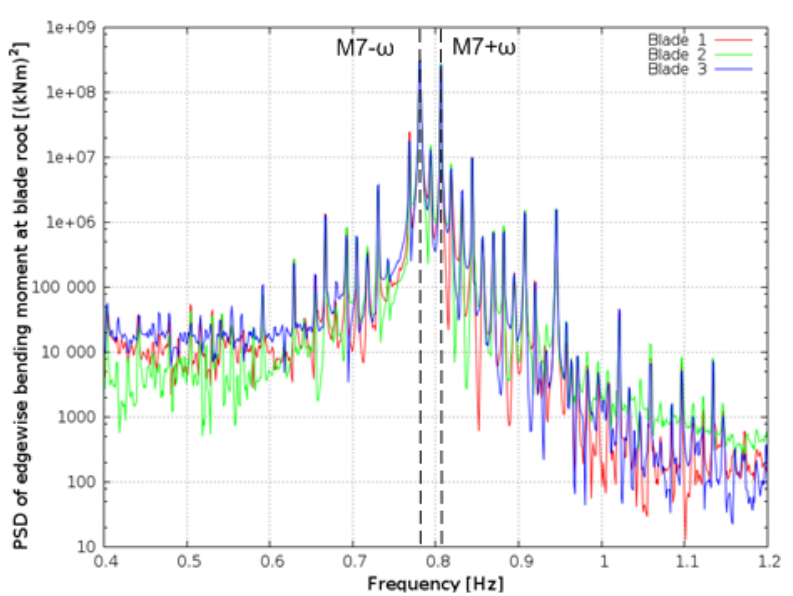

(b)

Figure 21. PSD of the edgewise bending moment at the blade root; $U=42.5 \mathrm{~m} \mathrm{~s}^{-1}$ and yaw $=30^{\circ}$. (a) Turbulent inflow (focus plot) and (b) uniform inflow (focus plot).

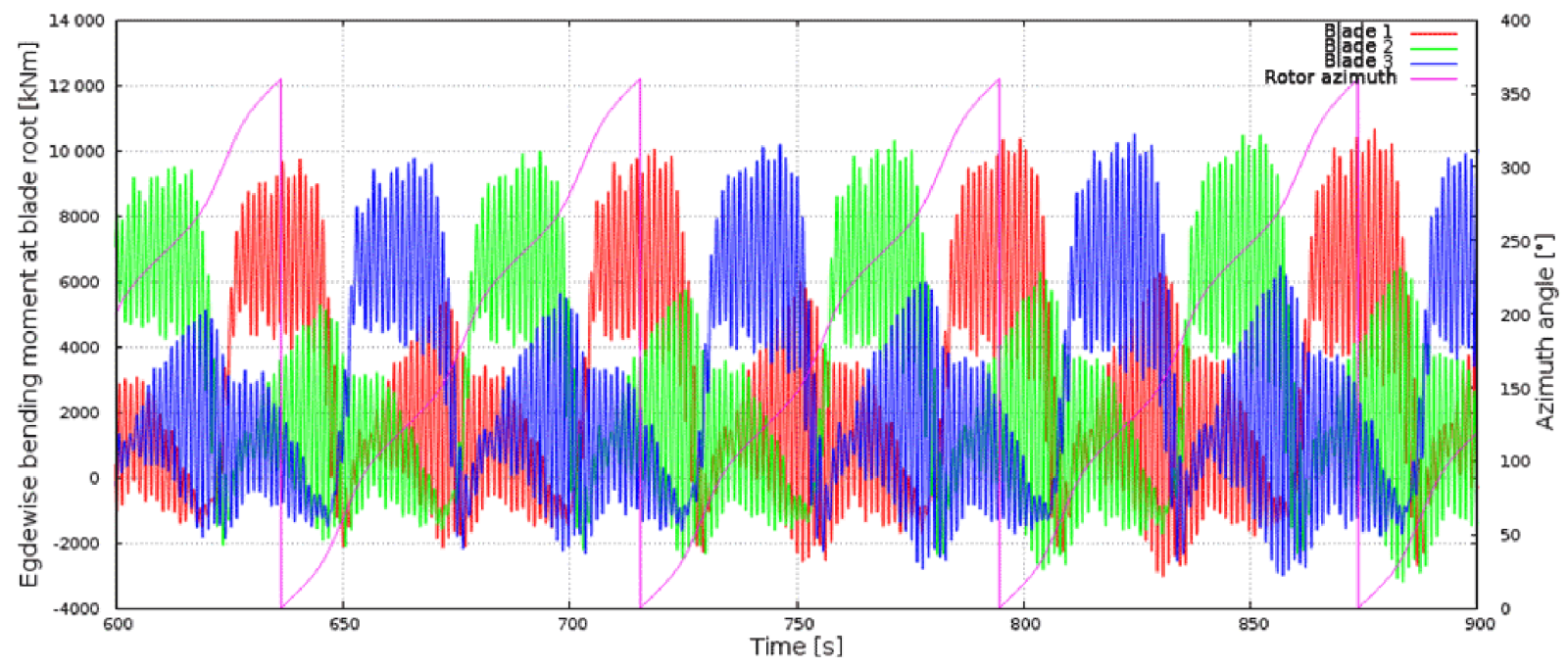

Figure 22. Time series of the edgewise bending moment at the blade root with uniform inflow at $U=42.5 \mathrm{~m} \mathrm{~s}^{-1}$ and $\mathrm{yaw}=30^{\circ}$.

three correspond to the three edgewise (out-of-plane) modes M5, M6 and M7. Again, the frequencies of the highly excited modes in the results of the time domain analysis agree well with the frequencies predicted by the stability tool (Fig. 19a) for the low damped rotor out-of-plane modes. The largest peak is seen at the frequency $0.8 \mathrm{~Hz}$ (the frequency of M7), indicating that this is indeed the lowest damped mode of the rotor. A closer look in the vicinity of the $0.8 \mathrm{~Hz}$ peak (see focus plot Fig. 21a) reveals two additional peaks around the center frequency of $0.8 \mathrm{~Hz}$ with a frequency shift of about $\pm 0.01 \mathrm{~Hz}$. The above frequency shift corresponds to the average idling rotational frequency ( $1 \mathrm{p}$ frequency). These peaks represent rotating periodic dynamics in the system (in terms of the principle eigenfrequency) and they are found to be even more excited than the center frequency peak. Such peaks are not observed in the PSD plot for the $0^{\circ}$ yaw case since the idling speed of the rotor is almost zero, as seen in Fig. 15. In a simulation with uniform inflow at the same wind speed $\left(42.5 \mathrm{~m} \mathrm{~s}^{-1}\right)$, the abovementioned peaks become more distinct (other peaks also appear that correspond to higher multiples) as seen in the PSD plot in Fig. 21b. It is noted that in the uniform inflow case, after an initial transient, the idling speed reaches a constant value $(0.78 \mathrm{RPM} ; 0.013 \mathrm{~Hz})$. This explains why the two peaks appear at frequencies exactly equal to $\pm \omega$ of the principle eigenvalue. In the case of turbulent wind, the idling speed continuously changes and therefore the obtained peaks are more spread. The amplifying response of the edgewise moment in the simulation with uniform inflow shown in Fig. 22 indicates that under uniform inflow conditions the system is unstable. The above result is in perfect agreement with the results of the eigenvalue analysis, which show that the M7 mode is negatively damped at 


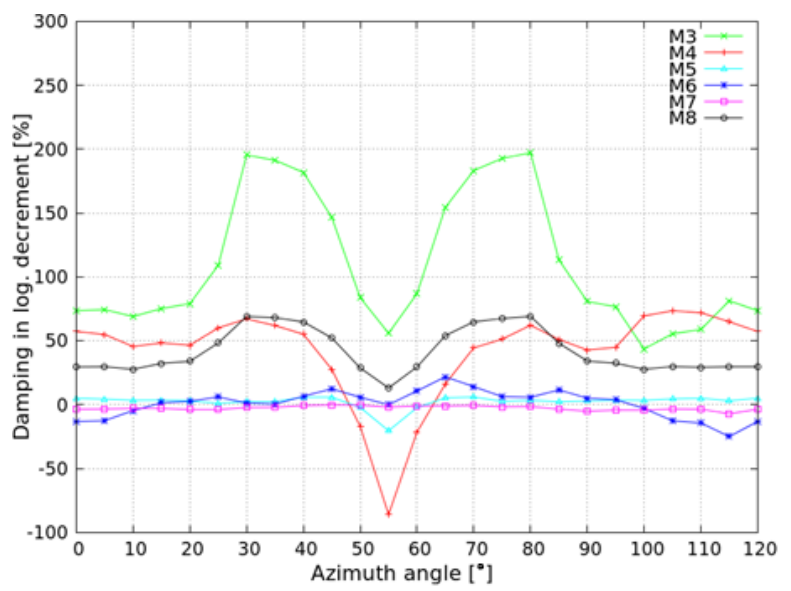

(a)

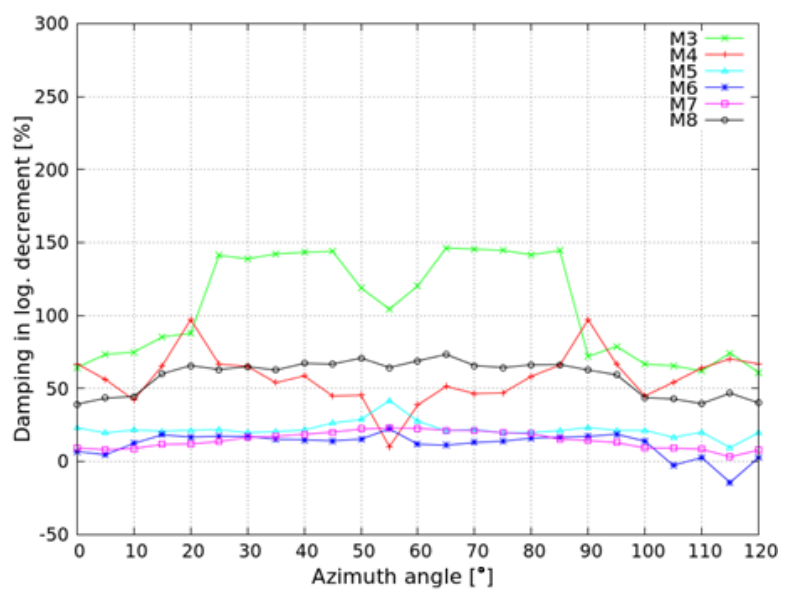

(b)

Figure 23. Modal damping of rotor modes versus azimuth angle at $U=42.5 \mathrm{~m} \mathrm{~s}^{-1}$ and yaw $=45^{\circ}$. (a) Steady-state aerodynamics and (b) unsteady aerodynamics (ONERA modeling).

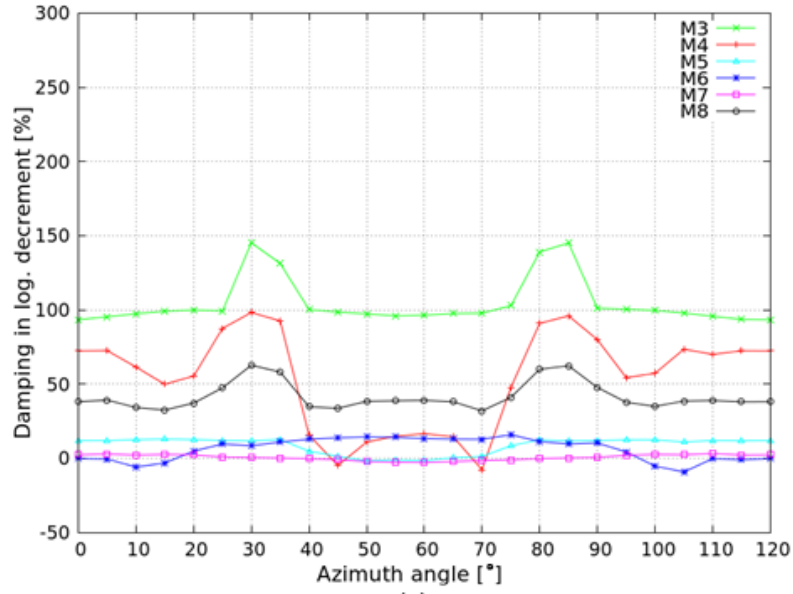

(a)

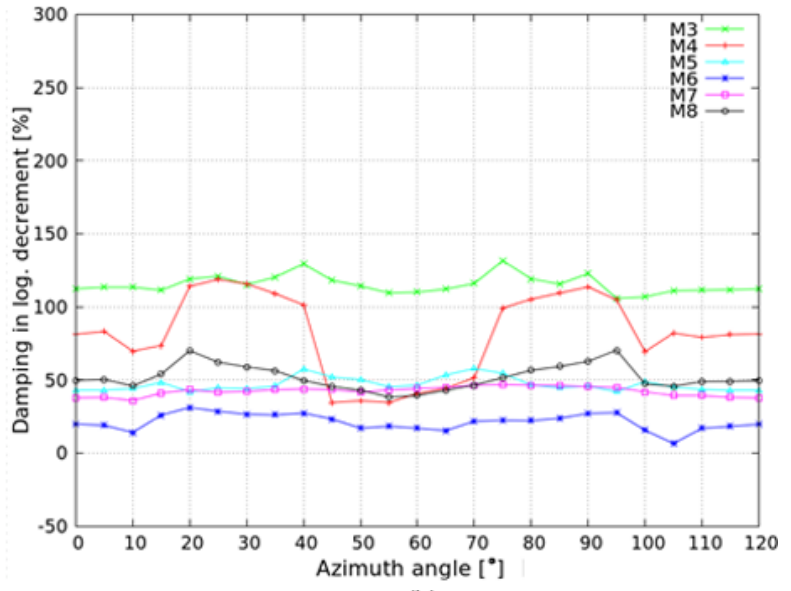

(b)

Figure 24. Modal damping of rotor modes versus azimuth angle at $U=42.5 \mathrm{~m} \mathrm{~s}^{-1}$ and yaw $=60^{\circ}$. (a) Steady-state aerodynamics and (b) unsteady aerodynamics (ONERA modeling).

all azimuth angles. In turbulent wind simulations, instabilities are mitigated as a result of the incoherent loading of the blades along their span and the continuous variation in the inflow that pushes the blade in and out of stall (regions with negative $C_{L}$ slope).

In Fig. 20a high energy levels are noted in the frequency range $[0.5 \mathrm{~Hz}, 0.6 \mathrm{~Hz}$. The peak corresponds to the in-plane mode M4. As discussed earlier, according to the stability predictions, this is the lowest damped flapwise mode. The smooth shape and the spread of the peak indicate the high aerodynamic damping involved.

In Figs. 23 and 24 the damping results for the 45 and $60^{\circ}$ yaw cases are shown. Results for both steady and unsteady aerodynamics are compared. For yaw angles higher than $30^{\circ}$ the system stability is gradually restored. This is in agreement with the results of the time domain analysis, which showed a gradual decrease in the edgewise loads beyond the $30^{\circ}$ yaw angle. At the yaw angle $45^{\circ}$ (see Fig. 23), the damping of M7 increases compared to the $30^{\circ}$ yaw case. The damping as predicted with steady-state aerodynamics is negative but very close to zero for all azimuth angles, while unsteady aerodynamic analysis always produces positive damping. The lowest damped mode with both steady and unsteady aerodynamics appears to be M6 (asymmetric edge vertical/yaw mode). Negative values are obtained in the azimuth range $\left[105^{\circ}, 120^{\circ}\right]$ (also in the range $\left[0^{\circ}, 10^{\circ}\right]$ with steady-state aerodynamics). The damping of M4 appears to have a negative dip at the $55^{\circ}$ azimuth angle in the steadystate results, which is smeared out in unsteady computations. Overall the damping of the in-plane modes seems to decrease as the yaw angle increases; however, it still remains highly positive over a wide range of azimuth angles. 

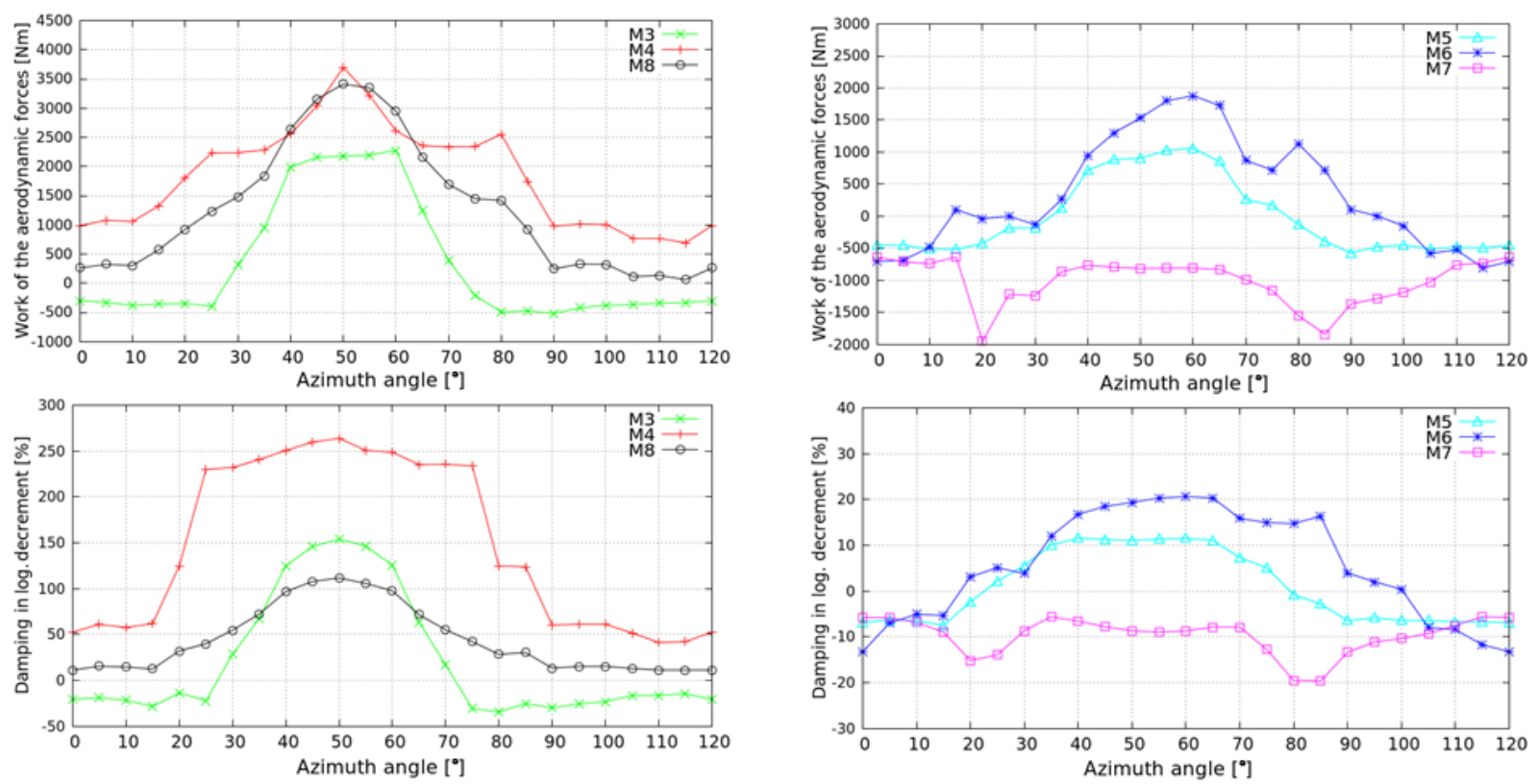

(a)

(b)

Figure 25. Comparison between aerodynamic work ( $0.2 \mathrm{~m}$ modal amplitude) and damping results versus azimuth angle for steady-state aerodynamics; $U=42.5 \mathrm{~m} \mathrm{~s}^{-1}$ and yaw $=30^{\circ}$. (a) In-plane (flapwise) modes and (b) out-of-plane (edgewise modes).

Damping results at the yaw angle $60^{\circ}$ using steady-state aerodynamics (see Fig. 24a) are found to be very similar to those for the $45^{\circ}$ yaw case. Unsteady results (see Fig. 24b) indicate that the damping of M7 continues to increase as the yaw angle increases. The same holds true with mode M6. It is noted that all out-of-plane modes are positively damped at $60^{\circ}$ of yaw, while M6 is the mode with the lowest damping characteristics.

\subsection{Work computation results}

The results of the eigenvalue analysis in the previous section are further evaluated by computations of the work done by aerodynamic forces in imposed harmonic vibrations. The blade is subjected to an externally imposed harmonic motion following the shape and frequency of the mode considered, and the unsteady aerodynamic loads are calculated along its span at various stations. Then the work done by the aerodynamic loads is computed over one cycle of the blade oscillation. The above work is directly associated with the damping of the corresponding mode. The mode shapes and eigenfrequencies used in the present analysis are the aeroelastic ones obtained through the eigenvalue analysis on the full aeroelastic system. As opposed to the structural mode shapes (obtained for the free vibration problem in vacuum conditions), the aeroelastic mode shapes also include the phase shift between the various components of the blade motion and therefore provide more a realistic prediction of the work distribution.
Work analysis is able to bring more insight into stability computations because it provides stability characteristics separately for each blade. When eigenvalue analysis produces a negative damping value for a specific mode, work analysis can identify which blade is responsible for the instability and the spanwise extent of the negative damping contribution. The nonlinear behavior of the aerodynamic loads can also be investigated by changing the amplitude of the forced oscillation. Since one of the main objectives of the present work is the evaluation of the linear eigenvalue analysis predictions, the modal amplitude was kept low and equal to $0.2 \mathrm{~m}$ at the blade tip. As in previous analyses, the analysis focuses on the yaw angle $30^{\circ}$ at which stall-induced vibrations mainly take place only on rotor modes.

In the present analysis, work computations have been performed using both ONERA and BL modeling. Since the linearized version of the BL model is not available in GAST_lin, the aeroelastic modes predicted by ONERA unsteady aerodynamics are employed in all work computations. Under the assumption that aeroelastic mode shapes are not significantly affected by different dynamic stall models, such a comparison is regarded as meaningful and can address differences between the two models in predicted unsteady hysteresis loops and resulting work distributions along the span of the blades.

In Figs. 25 and 26, the aerodynamic work results (integrated over the span and summed for the three blades) are compared against eigenvalue analysis results for both steadystate and unsteady aerodynamics. The cross-comparison of the two sets can only be performed at a qualitative level be- 

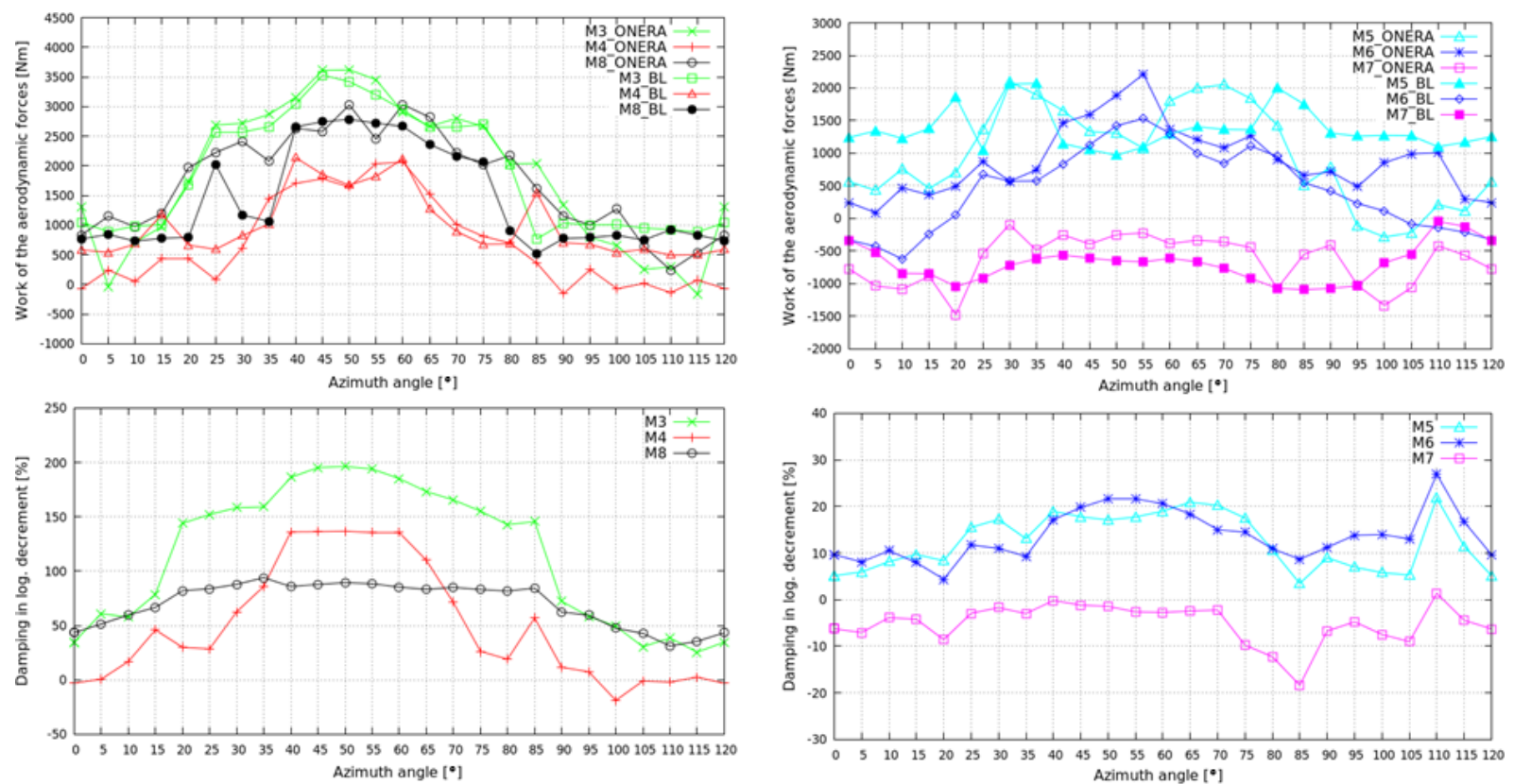

(a)

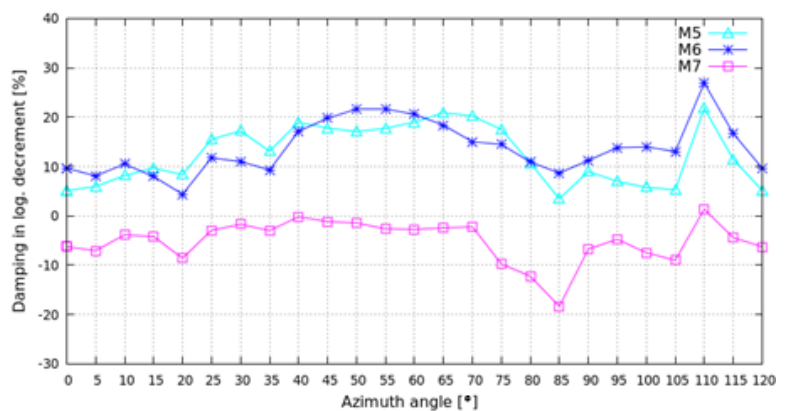

(b)

Figure 26. Comparison between aerodynamic work ( $0.2 \mathrm{~m}$ modal amplitude) and damping results versus azimuth angle for unsteady aerodynamics (ONERA and BL model); $U=42.5 \mathrm{~m} \mathrm{~s}^{-1}$ and yaw $=30^{\circ}$. (a) In-plane (flapwise) modes and (b) out-of-plane (edgewise modes).

cause in order to obtain the nonlinear damping of a particular mode through work computations, the predicted work value must be divided by the modal mass of the mode that is not the same for the different modes.

For steady-state aerodynamics (see Fig. 25) the agreement between work computations and eigenvalue analysis results is very good. Some differences are only noted in the shape of the work distribution for M4. Eigenvalue analysis predicts almost constant, highly positive damping of about $250 \%$ in the azimuth range $\left[25^{\circ}, 75^{\circ}\right]$. In the work results, despite the high work values obtained in the same azimuth range, a peak value is predicted at the azimuth angle $50^{\circ}$. As in the eigenvalue analysis, M8 remains positively damped at all azimuth angles, while $\mathrm{M} 3$ exhibits negative damping values in the azimuth ranges $\left[0^{\circ}, 25^{\circ}\right]$ and $\left[75^{\circ}, 120^{\circ}\right]$. Both methods agree that the damping of $\mathrm{M} 7$ is negative at all azimuth angles and that M5 and M6 are mostly positively damped.

For unsteady aerodynamics (see Fig. 26) and in regard to flap modes, a good correlation between work results and eigenvalue analysis results predicted with the ONERA model is obtained. Larger differences are noted in M3 but only at specific azimuth angles (for example, 5 and $115^{\circ}$ ). In these particular azimuth positions the eigenvalue analysis predicts positive damping, while in the work analysis damping is negative. When comparing work results of the two unsteady aerodynamic models (ONERA versus BL), reasonable agreement is again obtained. Larger differences are seen in M3 and M4 when the damping of the two modes drops. Overall the
BL model seems to predict higher minimum work for the above modes.

In regard to the edgewise modes, the results of the work analysis also compare well with the results of the eigenvalue analysis. The work calculated for mode $\mathrm{M} 7$ by the $\mathrm{ON}-$ ERA model remains negative over the whole azimuth range, whereas the same calculation for M5 and M6 produces positive work values. This is in line with the results of the eigenvalue analysis using the same dynamic stall model. When comparing the work results of the two unsteady aerodynamic models (ONERA versus BL), the largest differences are seen in M5. In the azimuth ranges $\left[0^{\circ}, 20^{\circ}\right]$ and $\left[80^{\circ}, 120^{\circ}\right]$, the ONERA model predicts lower work values that become negative in the azimuth range $\left[95^{\circ}, 105^{\circ}\right]$. On the other hand, the BL work predictions are shifted to slightly lower values for M6 and obtain negative values in the azimuth ranges $\left[0^{\circ}\right.$, $\left.15^{\circ}\right]$ and $\left[105^{\circ}, 120^{\circ}\right]$. In M7, both unsteady aerodynamic models agree that it is the lowest damped mode that obtains negative work values over the whole azimuth range.

It is noted that differences between eigenvalue analysis and work results in the shape of the curves are larger in the case of unsteady aerodynamics. Despite the relatively small amplitude of $0.2 \mathrm{~m}$ used in the work analysis, deviations from the eigenvalue analysis results are large in some cases. The explanation for the above differences is the strong nonlinearity of the ONERA equations, especially within the stall region. As described in Sect. 3.2, in the ONERA model, dynamic stall aerodynamic forces are derived through the solution of a set of first- and second-order time differen- 
tial equations with variable coefficients (coefficients depend on steady-state polars). In regions where the gradient of the steady-state polars changes rapidly, the linearized set of equations fails to correctly represent the actual nonlinear nature.

In Fig. 27 a snapshot of the shape of the negatively damped M7 mode is shown along with the tip-motion traces of the three blades. Clearly, the mode is asymmetric and out-ofplane with the two lower blades moving in one direction and the upper in the opposite direction. Modal motion resembles tilting of the rotor, and that is why the mode is called asymmetric out-of-plane tilt. It is seen that the three blades undergo coupled edgewise and flapwise motion (elliptical shape of modal displacement loops) with a different degree of coupling (indicated by the slope of the loops) and a different phase difference in the two motions (indicated by the width of the loops). All three blades follow an anticlockwise rotation (indicated by the symbol in the loops, which denotes the starting point on the loop). It is interesting to note that blade 3 essentially undergoes an in-plane motion (flapwise motion), while blade 1 presents the lowest degree of coupling with the flapwise direction.

In Figs. 28 and 29, work distributions along the three blades and $C_{L}$ hysteresis loops (unsteady simulation results) for the three blades at $r / R=0.90$ are shown for M7 and the azimuth angles 20 and $100^{\circ}$. Simulations have been performed using both unsteady aerodynamic models (ONERA and BL). For the above azimuth angles, the work calculations using the ONERA model predict the highest negative work values of the particular mode. In the abovementioned plots, $1 \mathrm{~m}$ of amplitude in the blade tip motion has been considered. Wider hysteresis loops are attained in this way and thereby the unsteady character of the flow is better illustrated. At $20^{\circ}$ rotor azimuth, both unsteady models agree that blade 2 (located at $140^{\circ}$ azimuth) provides the highest negative work. As seen in the $C_{L}-\mathrm{AOA}$ plot, blade 2 experiences $\mathrm{AOA}$ in the post-stall region at negative angles. In agreement with the results for the pitching airfoils presented in Sect. 3.2, the ONERA model predicts a wider dynamic stall loop for blade 2 . Negative work is also contributed by blade 1 (located at $20^{\circ}$ ). Blade 1 operates in the post-stall region at positive AOA. Again, the ONERA model predicts a wider loop than the BL model. The difference in the shape of the $C_{L}$-AOA loops between blade 1 and blade 2 (both in the post-stall region) is due to the different motions (in terms of flap-edge coupling and phase difference between the two directions of motion) undergone by the two blades in M7 (see Fig. 27). Blade 1 motion is dominated by vibrations in the edgewise direction with low coupling with the flapwise direction (about $20 \%$ ), while blade 2 exhibits a stronger flap-edge coupling. As a result of the lower flapwise component in blade 1 motion, the range of AOA variation seen by blade 1 is low. The large width of the blade 1 loop can be explained by the relatively highly reduced frequency of the motion, which is about $k=0.14$ for this specific case
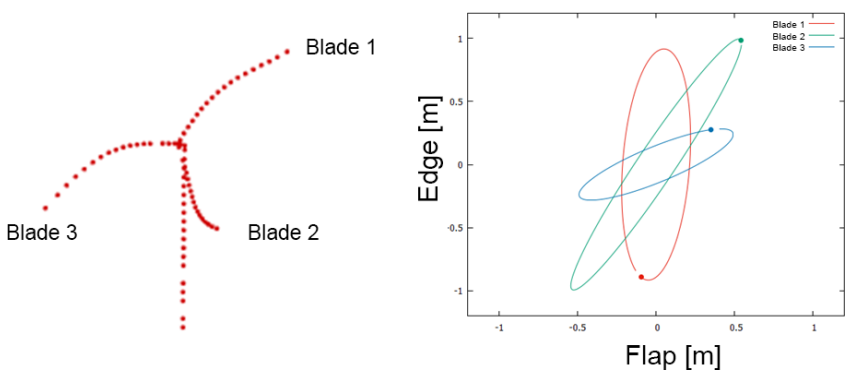

Figure 27. Shape of the lowest damped M7 mode at the azimuth angle $20^{\circ}$. Trace of the modal displacement of the three blades.

(frequency $0.8 \mathrm{~Hz}$; chord at $80 \mathrm{~m}$ span, $2.3 \mathrm{~m}$; wind velocity $42.5 \mathrm{~m} \mathrm{~s}^{-1}$ ). Comparing the work predictions of the two unsteady models reveals relatively good agreement. Some differences are noted in the mid-span of blade 1 for which the ONERA model predicts higher work values. The airfoil sections of the mid-span experience AOA in the vicinity of the $C_{L \max }$ angle, and therefore the higher hysteresis predicted by the ONERA model enhances the damping of the respective part of the blade. At $100^{\circ}$ rotor azimuth according to the ONERA model, negative work is almost evenly contributed by blades 2 and 3 (located at 220 and $340^{\circ}$, respectively). The shapes of the $C_{L}-\mathrm{AOA}$ loops for blade 1 are quite different from the shapes for blades 2 and 3 . This is mainly because of the different flow conditions encountered by the different blades. Blades 2 and 3 operate in deep stall (well within the negative $C_{L}$-AOA slope region); while blade 1 encounters light stall conditions (mainly positive slopes up to the $\left.C_{L \min }\right)$. The BL model predicts considerably lower negative work values along the span of blade 2 compared to the ONERA model. As seen in the loop plot, there is a distinct difference in the slopes of the loops provided by the two models. The BL loop clearly has a higher slope, justifying the higher work values predicted by the model. On blade 1, the BL model predictions agree with the ONERA results that the loop obtains a positive slope. However, the width of the loop predicted by the BL model is narrower. Similar loops and work distributions are predicted by both models on blade 3 .

It is also noted that the results of the work analysis seem to agree well with the results of the time domain simulations in turbulent inflow (depicted in Fig. 13). In both sets at the rotor azimuth of $20^{\circ}$, the blade with an azimuth angle of $20^{\circ}$ is in the post-stall region at positive AOA. The blade with azimuth angle $140^{\circ}$ is facing stall at negative AOA, and the blade with azimuth angle $260^{\circ}$ is also experiencing negative AOA but in the attached flow region. Similarly for the rotor azimuth of $100^{\circ}$, the blade positioned at a $100^{\circ}$ angle is experiencing negative AOA close to the $C_{L \text { min }}$ angle, while the other two blades operate in deep stall at negative and positive AOA. 


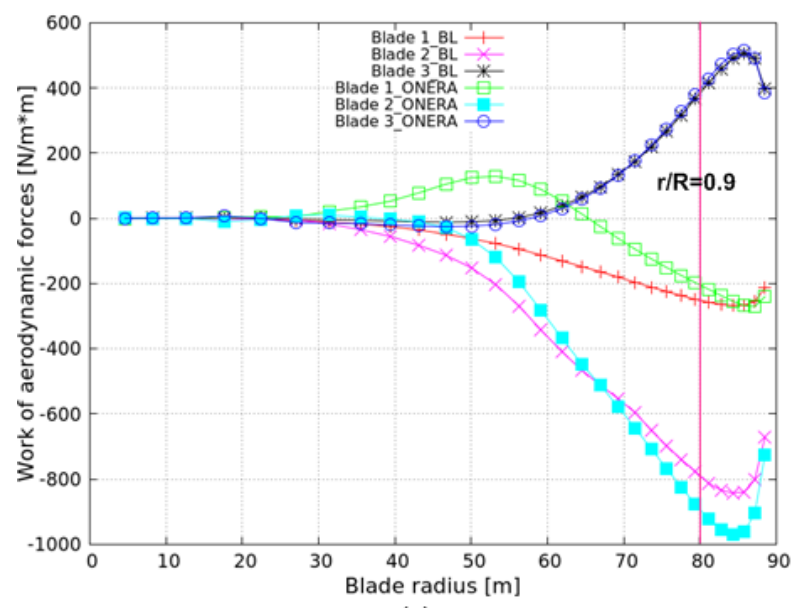

(a)

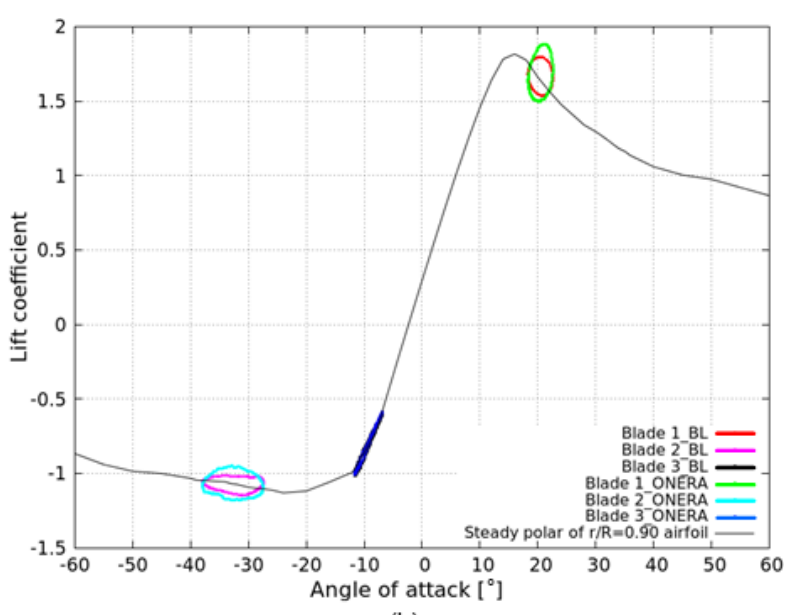

(b)

Figure 28. (a) Distribution of aerodynamic work over the three blades for mode M7. Azimuth angle $20^{\circ}$. (b) $C_{L}-\mathrm{AOA}$ loops of the three blades at $r / R=0.90$ for mode M7. Azimuth angle $20^{\circ}$.

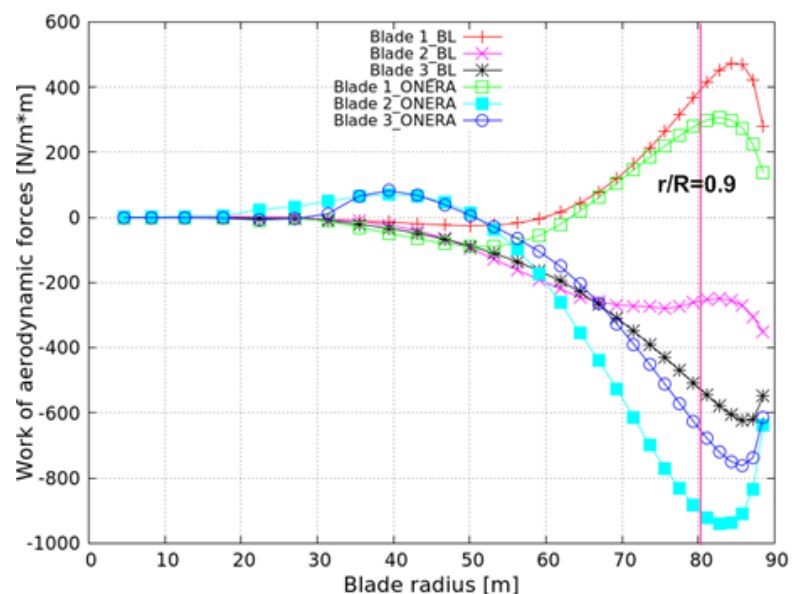

(a)

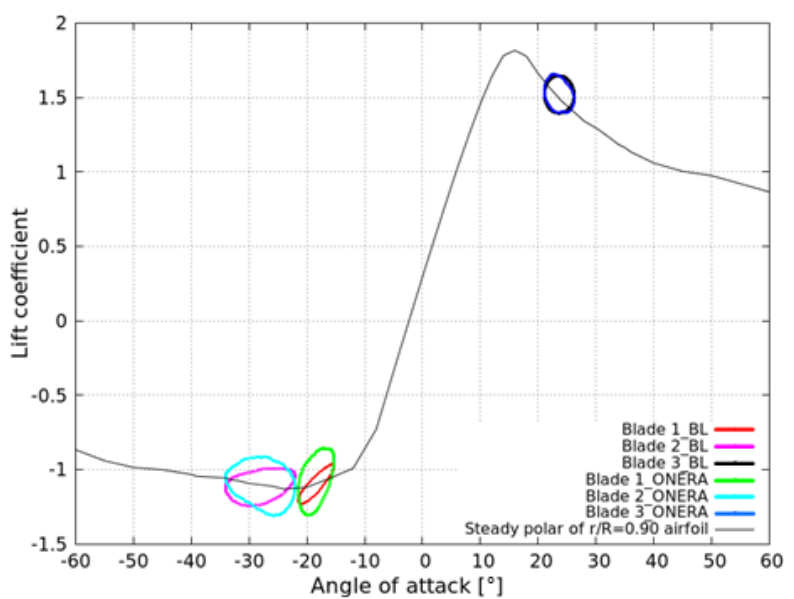

(b)

Figure 29. Distribution of aerodynamic work over the three blades for mode M7. Azimuth angle $100^{\circ}$. (b) $C_{L}-\mathrm{AOA}$ loops of the three blades at $r / R=0.90$ for mode M7. Azimuth angle $100^{\circ}$.

\section{Conclusions}

The aeroelastic stability characteristics of the DTU $10 \mathrm{MW}$ reference wind turbine in standstill or slowly idling operation have been numerically analyzed. To this end, a consistent and computationally cost effective modeling environment has been presented. It is composed of the core eigenvalue stability analysis tool GAST_lin supported by the nonlinear time domain aeroelastic analysis code hGAST and a nonlinear stability analysis tool in which damping is assessed through computations of the aerodynamic work under imposed periodic motion. The predictions of the eigenvalue tool are evaluated through comparisons with the results of both nonlinear tools. It is a model-based validation of the linearized model against its nonlinear counterparts. The crosscomparison of the above tools demonstrated that fast linear eigenvalue stability tools can be used as a basis for characterizing the stability of turbines in idling operation.

It is noted that the proposed eigenvalue stability analysis cannot always provide a definite answer to the question of whether the system has adequate damping or not. An example is when a mode is positively damped in one part of the revolution and negatively damped in another. However, this definite answer is also difficult even through Floquet analysis (or any other type of analysis). This is first because of the strong nonlinearity of the aerodynamics in dynamic stall, but most importantly because of the effect that turbulent wind has on the behavior of the system. It is noted that turbulent wind cannot be easily accounted for in any stability analyses. An example of such uncertainty is given in the paper. Under uniform inflow conditions (wind speed $42.5 \mathrm{~m} \mathrm{~s}^{-1}$ and yaw $30^{\circ}$ ) the system is unstable, as shown in Fig. 22. Vibrations 
continuously grow in time and lead to divergence after a certain time. This is in agreement with the eigenvalue analysis results, which predict that M7 remains negatively damped throughout the revolution. Also, the frequency of the predicted negatively damped mode M7 agrees with the PSD of the edgewise moment, which exhibits a peak at the frequency $0.8 \mathrm{~Hz}$. For the same case and for turbulent inflow conditions, vibrations do not lead to critical instabilities in the system. Amplifying edgewise vibrations are observed in part of the time series in Fig. 13, which after a certain time decay. This is because, as a result of the turbulent wind, operation moves continuously in and out the negative $C_{L}$ slope region as indicated in the $C_{L}-\mathrm{AOA}$ loops in Fig. 14. So, the eigenvalue tool should not be considered a stand-alone tool for assessing the stability behavior of an idling turbine. It should be used in combination with nonlinear tools. The aim of the proposed computational environment is to identify conditions that favor instabilities that could be the starting point for improving blade structural dynamics and/or aerodynamics.

The analysis showed that the lowest damped modes of the $10 \mathrm{MW}$ idling rotor are the out-of-plane modes (symmetric and asymmetric). At a yaw misalignment of $30^{\circ}$, the asymmetric out-of-plane tilt mode attains negative damping throughout the entire range of azimuth angles. At higher yaw angles, the stability of the rotor is gradually restored and the damping of the out-of-plane modes returns to positive values. At the yaw angle $30^{\circ}$, the maximum edgewise loads in the time domain analysis are also obtained. At the above conditions, instabilities are noted in the results of the time domain analysis, which through FFT on loads are identified to be linked to the negatively damped out-of-plane tilt mode. Although the instabilities seen in the results of the time domain analysis do not continuously grow as suggested by the negative damping of the eigenvalue predictions, they lead to some quite severe stall-induced vibrations in the edgewise direction. The less coherent loading conditions developing over the rotor disk under turbulent inflow lead to mitigated vibrations compared to the uniform inflow case considered in the stability analysis. As a result of the temporal and spatial variation in the wind, the blades are continuously pushed in and out of the negative $C_{L}$ slope regions, and therefore vibrations do not have enough time to build up. At higher yaw angles, edgewise loads decrease in agreement with the results of the eigenvalue analysis, which predicts positive damping values of the out-of-plane modes at yaw angles higher than $30^{\circ}$.

Eigenvalue stability analyses are performed for both steady-state and unsteady aerodynamics. The aim of running simulations, also for steady-state aerodynamic conditions, is to explore the range of damping predictions, especially in connection to the fact that many of the-state-of-the art engineering dynamic stall models automatically switch to a steady state at very high AOA. Results indicate that steadystate analysis is more conservative, providing higher negative damping values for all out-of-plane modes. Overall the agreement of the eigenvalue stability analysis with the work computations is good. Some larger differences are noted at specific azimuth positions when unsteady aerodynamics is employed. The differences are mainly due to the strong nonlinearity of the ONERA equations particularly triggered in deep stall conditions.

Data availability. The measured datasets for the pitching airfoils are the property of DTU. They can be found in the reports cited in the paper. Please contact the corresponding author in order to obtain the rest of the data presented in the paper.

Competing interests. The authors declare that they have no conflict of interest.

Special issue statement. This article is part of the special issue "The Science of Making Torque from Wind (TORQUE) 2016" as a result of an annual international conference with the same name held in Munich, Germany from 5 to 7 October 2016.

Acknowledgements. The work presented in this paper was partially funded by the European Commission Seventh Framework Programme under grant agreements FP7-ENERGY-2012-12STAGE-308974 (INNWIND.EU) and FP7-ENERGY-20131/no.608396 (AVATAR).

Edited by: Carlo L. Bottasso

Reviewed by: Morten H. Hansen and Stefano Cacciola

\section{References}

Bak, C., Fuglsang, P., Johansen, J., and Antoniou, I.: Wind Tunnel Tests of the NACA 63-415 and a Modified NACA 63-415 Airfoil, Ris $\varnothing-\mathrm{R}-1193(\mathrm{EN})$, Ris $\varnothing$ National Laboratory, Roskilde, Denmark, 2000.

Bak, C., Zahle, F., Bitsche, R., Kim, T., Yde, A., Henriksen, L. C., Natarajan, A., and Hansen, M. H.: Description of the DTU 10MW Reference Wind Turbine, DTU Wind Energy Report-I0092, 2013.

Bottasso, C. and Cacciola, S.: Model independent periodic stability analysis of wind turbines, Wind Energy, 18, 865-887, 2015.

Coleman, R. P. and Feingold, A. M.: Theory of self excited mechanical oscillations of helicopter rotors with hinged blades, Technical Report NACA-TN-3844, NACA-TR-1351, Langley Research Center, 1958.

Fuglsang, P., Antoniou, I., Dahl, K. S., and Madsen, H. A.: Wind Tunnel Tests of the FFA-W3-241, FFA-W3-301 and NACA 63430 airfoils, Ris $\varnothing-\mathrm{R}-1041(\mathrm{EN})$, Ris $\varnothing$ National Laboratory, Roskilde, Denmark, 1998.

Hansen, M. H.: Improved Modal Dynamics of Wind Turbines to Avoid Stall-Induced Vibrations, Wind Energy, 6, 179-195, 2003.

Hansen, M. H.: Aeroelastic instability problems for wind turbines, Wind Energy, 10, 551-577, 2007. 
Hansen, M. H., Gaunaa, M., and Madsen, H. A.: A BeddoesLeishman type dynamic stall model in state-space and indicial formulation, Risoe Report, Risoe-R-1354(EN), 2004.

Heinz, J. C., Sørensen, N. N., Zahle, F., and Skrzypiński, W.: Vortex-induced vibrations on a modern wind turbine blade, Wind Energy, 19, 2041-2051, https://doi.org/10.1002/we.1967, 2016.

Manolas, D. I., Riziotis, V. A., and Voutsinas, S. G.: Assessing the importance of geometric nonlinear effects in the prediction of wind turbine blade loads, Computational and Nonlinear Dynamics Journal, 10, 041008, 1-15, 2015.

Petersen, J. T., Madsen, H. A., Bjorck, A., Enevoldsen, P., Øye, S., Ganander, H., and Winkelaar, D.: Prediction of Dynamic Loads and Induced Vibrations in Stall, Ris $\varnothing-\mathrm{R}-1045(\mathrm{EN})$, Ris $\varnothing \mathrm{Na}-$ tional Laboratory, Roskilde, 1998.

Petot, D.: Differential Equation Modeling of Dynamic Stall, Recherché Aerospatiale, 5, 59-72, 1989.

Pirrung, G., Madsen, H. A., and Schreck, S.: Trailed vorticity modeling for aeroelastic wind turbine simulations in standstill, Proceedings of the Science of Making Torque from Wind Conference, TORQUE 2016, 5-7 October, Munich, Germany, 2016.

Politis, E. S., Chaviaropoulos, P. K., Riziotis, V. A., Voutsinas, S. G., and Romero-Sanz, I.: Stability analysis of parked wind turbine blades, Proceedings of the EWEC 2009, Scientific Track, 1619 March, Marseille, France, 2009.

Riziotis, V. A.: Aeroelastic analysis of stall on wind turbine rotors, $\mathrm{PhD}$ thesis, National Technical University of Athens, Athens, Greece, 2003.

Riziotis, V. A., Voutsinas, S. G., Politis, E. S., and Chaviaropoulos, P. K.: Aeroelastic stability of wind turbines: the problem the methods and the issue, Wind Energy, 7, 373-392, 2004.
Shi, L., Riziotis, V. A., Voutsinas, S. G., and Wang, J.: A consistent vortex model for the aerodynamic analysis of vertical axis wind turbines, J. Wind Eng. Ind. Aerod., 135, 57-69, 2014.

Skrzypiński, W. and Gaunaa, M.: Wind turbine blade vibration at standstill conditions - the effect of imposing lag on the aerodynamic response of an elastically mounted airfoil, Wind Energy, 18, 515-527, 2015.

Skrzypiński, W., Gaunaa, M., Sørensen, N., Zahle, F., and Heinz, J.: Self-induced vibrations of a DU96-W-180 airfoil in stall, Wind Energy, 17, 641-655, 2014a.

Skrzypiński, W., Gaunaa, M., Sørensen, N., Zahle, F., and Heinz, J.: Vortex-induced vibrations of a DU96-W-180 airfoil at $90^{\circ}$ angle of attack, Wind Energy, 17, 1495-1514, 2014b.

Skrzypiński, W., Gaunaa, M., and Heinz, J.: Modeling of vortexinduced loading on a single blade installation-setup, The Science of Making Torque from Wind Conference, TORQUE 2016, Munich, Germany, 5-7 October, Journal of Physics: Conference series 753, 082037, 2016.

Skjoldan, P. F. and Hansen, M. H.: On the similarity of the Coleman and Lyapunov - Floquet transformations for modal analysis of bladed rotor structures, J. Sound Vib., 327, 424-439, 2009.

Wang, K., Riziotis, V. A., and Voutsinas, S. G.: Aeroelastic Stability of Idling Wind Turbines, Proceedings of the Science of Making Torque from Wind Conference, TORQUE 2016, 5-7 October, Munich, Germany, 2016.

Zou, F., Riziotis, V. A., Voutsinas, S. G., and Wang, J.: Analysis of vortex and stall induced vibrations at standstill conditions using a free wake aerodynamic code, Wind Energy, 18, 2145-2169, 2015 . 